\title{
On an Unsatisfiability-satisfiability Prover
}

\author{
By
}

\section{Chiharu Hosono*}

\section{$\S 1$. Introduction}

In recent decades the study of automated theorem proving has become to be accelerated with the progress of the computers. Therefore many proving methods have been implemented, for example [1], [4], [6] or theoretically investigated, for example [2], [5]. Among them most provers take their base from Robinson's resolution method. By the well known fact that the first order logic is undecidable, the most effort goes to the improvement of the efficiency of the successful test of a theorem, but not to widen the decidability of prover. In this paper we study a new unsatisfiability-satisfiability prover and its basic properties. Our main results, besides the presentation of our prover, is to show that our procedure always terminates if a given set of clauses is satisfiable in some finite domain. The unsatisfiability checking of our procedure is as strong as the existing complete proof procedures, so we examine the strength of the satisfiability checking part of our procedure in $\S 5$ and $\S 6$.

In $\S 2$ we define several basic concepts and show a number of propositions related to them. In $\S 3$ we give an unsatisfiability-satisfiability prover, and show its soundness that is for any unsatisfiable set of clauses the procedure says that it is unsatisfiable, and if the procedure says that a set of clause is satisfiable, then it is in fact satisfiable. In $\S 4$ we show a number of basic properties of the procedure. In $\S 5$, we show that for a given set of clauses assumed to be satisfiable in a simple theory of Herbrand universe, the procedure terminates saying it is satisfiable. In $\S 6$ we show that for a given set of clauses assumed to be satisfiable in some finite domain, the procedure terminates saying it is satisfiable. In $\S 7$ we discuss the results in this paper.

\section{§2. Preliminaries}

In this section we define some basic concepts and we show several propositions related to these. It is well known that any first order formula can be transformed to a universal formula (or a clausal form) using Skolem's functions

Communicated by S. Takasu, June 20, 1988.

* Institute of Information Sciences and Electronic, University of Tsukuba, Ibaraki 305, Japan. 
not changing unsatisfiability. Hence to test a formula, we first negate it, then transform it to a clausal form, and test its unsatisfiability. For details of them refer to [3]. In the sequel we assume that the given formula is in the universal form and use the term of set of clauses for formula.

Definition 2.1. For a set $S$ of clauses, $\boldsymbol{L}_{\boldsymbol{P}}(S), \boldsymbol{L}_{C}(S)$ and $\boldsymbol{L}_{\boldsymbol{F}}(S)$ denote the set of all predicate symbols, all constant symbols and all function symbols of $S$ respectively.

It is noted that $\boldsymbol{L}_{P}(S), \boldsymbol{L}_{C}(S), \boldsymbol{L}_{\boldsymbol{F}}(S)$ are finite, and we assume these are non-empty. We use variable symbols $x, y, z, \cdots, x_{1}, \cdots$, etc., and predicate symbols $P, Q, \cdots$, etc. We do not use meta-variables. Hence simple variables may be considered as meta-variables. Also we may use the term of predicate for the term of predicate symbol, etc.

We review some terminologies. For a given set $S$ of clauses, terms that are constructed from symbols of $\boldsymbol{L}_{\boldsymbol{C}}(S)$ and $\boldsymbol{L}_{\boldsymbol{F}}(S)$, and height $(t)$ for a term $t$ are defined by recursion as the following:

Definition 2.2. Any constant $c \in L_{C}(S)$ and any variable are terms, and each height of them is 1 . For an $n$-ary function symbol $f \in \boldsymbol{L}_{F}(S)$ and terms $t_{1}, \cdots, t_{n}, f\left(t_{1}, \cdots, t_{n}\right)$ is a term where $f\left(t_{1}, \cdots, t_{n}\right)$ is understood to be a sequence of symbols as they stand, and height $\left(f\left(t_{1}, \cdots, t_{n}\right)\right)$ is $\max \left(\right.$ height $\left(t_{1}\right), \cdots$, height $\left.\left(t_{n}\right)\right)+1$.

Definition 2.3. For an $n$-ary predicate symbol $P \in L_{P}(S)$ and terms $t_{1}, \cdots, t_{n}$, $P\left(t_{1}, \cdots, t_{n}\right)$ is a prime formula or an atom simply. The height of $P\left(t_{1}, \cdots, t_{n}\right)$ is $\max$ (height $\left(t_{1}\right), \cdots$, height $\left.\left(t_{n}\right)\right)$, and if $n=0$, i. e. $P$ is a propositional variable, then height $(P)$ is 0 .

Definition 2.4. $\neg$ is the negation symbol. $L$ is called a literal if and only if $L$ is either an atom or $L$ is a negation of an atom. The height $(\neg A)$ is height $(A)$ for an atom $A$. For a literal $L, \sim L$ denotes the negation of it, i. e. $\sim L$ is $\neg A$ if $L$ is $A$, and $\sim L$ is $A$ if $L$ is $\neg A$ where $A$ is an atom.

Definition 2.5. $A$ clause $C$ is a finite set of literals. $A$ set $S$ of clauses means usually a finite set of clauses. The height of a clause and that of a set of clauses are the maximum height of those elements.

It is noted that a clause intuitively means the disjunction of literals, free variables are considered to be universally quantified for each clause, and a set of clauses means the conjunction of clauses. The satisfiability and the unsatisfiability are naturally defined. If a clause $C$ contains both $L$ and $\sim L$, then $C$ is said to be a tautology. $A$ clause $\left\{L_{1}, \cdots, L_{n}\right\}$ is also denoted by $L_{1} \vee \cdots \vee L_{n}$, and also $\left\{\sim L_{1}, \cdots, \sim L_{n}, L_{1}{ }^{\prime}, \cdots, L_{m}{ }^{\prime}\right\}$ is denoted by $L_{1} \wedge \cdots \wedge L_{n} \supset L_{1}{ }^{\prime} \vee \cdots \vee L_{m}{ }^{\prime}$. 
For clauses $C_{1}$ and $C_{2}, C_{1} \vee C_{2}$ denotes $C_{1} \cup C_{2}$.

A substitution $\theta$ is as usual a simultaneous substitution of terms for variables. For example $\{c / x, f(c, c) / y\}$ is a substitution where $c$ and $f(c, c)$ are substituted for $x, y$ respectively. Let $C$ be a clause, then $C^{\theta}$ denotes the clause obtained from $C$ substituted by $\theta$. A ground clause is a clause which has no occurrence of variables, and a ground instance is a substituted result which is a ground clause.

Definition 2.6. A contradictory instance of a set $S$ of clauses is a unsatisfiable set of ground instances of clauses of $S$.

For example the contradictory instance of $\{\{P(f(c, c))\},\{\neg P(x)\}\}$ is $\{\{P(f(c, c))\},\{\neg P(f(c, c))\}\}$. The well known Herbrand's theorem is that if a set $S$ of clauses is unsatisfiable, then there exists at least one contradictory instance of $S$.

Definition 2.7. The rank of a contradictory instance is the maximum height of atoms occurring in the instance, and the rank of $S$ is the minimum rank of all contradictory instances of $S$ and is denoted by $\operatorname{rank}(S)$.

It is noted that $\operatorname{rank}(S)=0$ if and only if $S$ includes an unsatisfiable subset of propositional clauses. For example $\operatorname{rank}(\{\{P(f(c, c))\},\{\neg P(x)\}\})=2$, and $\operatorname{rank}(\{\{P\},\{\neg P\},\{Q(f(c, c))\},\{\neg Q(x)\}\})=0$.

Definition 2.8. For a set $S$ of clauses, if $S^{\theta}$ is unsatisfiable where $\theta$ is $\left\{c_{x} / x \mid x\right.$ occurs in $\left.S\right\}$ and $c_{x}$ is a new distinct constant for $x, S$ is said to be a nominal contradiction.

For example $\{\{P(x)\},\{\neg P(x)\}\}$ is a nominal contradiction, but not $\{\{P(x)\},\{\neg P(y)\}\}$. It is noted that a contradictory instance is a nominal contradiction.

Definition 2.9. For a substitution $\theta, \theta$ is flat if and only if any term to be substituted in $\theta$ is a constant or a variable.

For example $\{c / x, u / y, v / z\}$ is a flat substitution.

Definition 2.10. For clauses $C$ and $C^{\prime}, \phi\left(C^{\prime}, C\right)$ denotes the clause $C^{\prime}-C$.

For example $\phi(\{P, Q, \neg R\},\{P, R\})$ is $\{Q, \neg R\}$.

Definition 2.11. A clause $C$ is subsumed by $C_{1}, \cdots, C_{n}$ if and only if there exist some substitutions $\theta_{1}, \cdots, \theta_{n}$ and $\left\{\psi\left(C_{1}{ }^{\theta_{1}}, C\right), \cdots, \phi\left(C_{n}{ }^{\theta_{n}}, C\right)\right\}$ is a nominal contradiction. If all $\theta_{1}, \cdots, \theta_{n}$ are flat, then a clause $C$ is flatly subsu'ned by 
$C_{1}, \cdots, C_{n}$, and if $n=1$, then a clause $C$ is simply subsumed by $C_{1}$. Also for 'sets $S, S^{\prime}$ of clauses, if any clause $C$ in $S$ is a tautology or subsumed by some clauses $C_{1}, \cdots, C_{n}$ in $S^{\prime}$, then $S$ is subsumed by $S^{\prime}$. The flat subsumption and the simple subsumption between $S$ and $S^{\prime}$ are defined similarly, and $S \ll S^{\prime}$ or $S^{\prime} \gg S$ denotes that $S^{\prime}$ is simply subsumed by $S$.

It is noted that in the definition 2.11, there may be same clauses in $C_{1}, \cdots, C_{n}$, so that the definition is somewhat ambiguous but the rigorous definition leads to a cumbersome notations and reduces the readability, and therefore we use this definition. If $C$ is subsumed by $C_{1}, \cdots, C_{n}$ then $C$ is a logical consequence of $C_{1}, \cdots, C_{n}$. We restricts the number of use of variants of $C^{\prime}$ within the number of occurrences $C^{\prime}$ in $C_{1}, \cdots, C_{n}$. Therefore the check of whether $C$ is subsumed by $C_{1}, \cdots, C_{n}$ or not, is effectively computable. For example $\neg P(y) \vee P(f(f(y, y), f(y, y)))$ is not subsumed by $\neg P(x) \vee P(f(x, x))$ but subsumed by $\neg P(x) \vee P(f(x, x))$ and $\neg P(x) \vee P(f(x, x))$ using the substitutions $\theta_{1}=\{y / x\}$ and $\theta_{2}=\{f(y, y) / x\}$ where the nominal contradiction is $\{\{\neg P(f(y, y))\},\{P(f(y, y))\}\}$.

Proposition 2.12. If a set $S$ of clauses is simply subsumed by a set $S^{\prime}$ of clauses and $S$ is unsatisfiable, then $\operatorname{rank}\left(S^{\prime}\right) \leqq \operatorname{rank}(S)$.

Proof. For a contradictory instance $X$ of $S$, there is a set of ground instances $X^{\prime}$ of $S^{\prime}$ which simply subsumes $X$. Hence $X^{\prime}$ is contradictory. By the definition of the simple subsumption, we can assume that any atom of $X^{\prime}$ occurs in $X$. Hence, obviously $\operatorname{rank}\left(X^{\prime}\right) \leqq \operatorname{rank}(X)$.

Definition 2.13. For a clause $C$, a substitution $\varepsilon$ is said to be a decomposition of $C$ if and only if all the variables of $C$ are substituted and the term to be substituted for each variable $x$ is either a constant $c \in \mathbb{L}_{C}(S)$ or a term $f\left(u_{1}, \cdots, u_{n}\right)$, where $\left\langle u_{1}, \cdots, u_{n}\right\rangle$ is a sequence of new distinct variables for $x$ and $f \in L_{F}(S)$. The number of decompositions of a clause is finite except the choice of $\left\langle u_{1}, \cdots, u_{n}\right\rangle$.

Definition 2.14. For an atom $P\left(t_{1}, \cdots, t_{n}\right)$ where $t_{i}$ is not a variable for any $i$, we define a predicate-decomposition operation $*$ of predicate symbol, for example an atom $P\left(c, f\left(t_{1}, t_{2}\right), g\left(s_{1}, s_{2}\right)\right)$ is rewritten as $P_{c f g}\left(t_{1}, t_{2}, s_{1}, s_{2}\right)$ introducing a new predicate symbol $P_{c f g}$ and removing the top level occurrences of $c, f$ and $g$. We extend this operation canonically to literals, clauses and sets of clauses, and the results of decompositions are denoted by $L^{*}, C^{*}$ and $S^{*}$, respectively.

This predicate-decomposition operation is only used in combination with decomposition operations as in the following definition. 
Definition 2.15. For a clause $C$ which has $n$ free variables, we define the expansion $C^{\uparrow}$ of $C$ by $C^{\uparrow}=\left\{C^{\varepsilon_{i} *} \mid 1 \leqq i \leqq N\right\}$ where the set $\left\{\varepsilon_{i} \mid 1 \leqq i \leqq N\right\}$ consists of all decompositions of $C$. If $C$ contains no free variable, then $C^{\uparrow}=\left\{C^{*}\right\}$. For a set $S$ of clauses, the expansion is $S^{\wedge}=\cup\left\{C^{\uparrow} \mid C \in S\right\}$.

For example let $S$ be $\{\{P(f(c, c))\},\{\neg P(x)\}\}$, then $S^{\uparrow}$ is $\left\{\left\{P_{f}(c, c)\right\},\left\{\neg P_{c}\right\}\right.$, $\left\{\neg P_{f}(x, y)\right\}$. It is noted that $\operatorname{rank}\left(S^{\uparrow}\right)=1, \operatorname{rank}(S)=2$ and $\boldsymbol{L}_{P}(S)$ and $\boldsymbol{L}_{P}\left(S^{\uparrow}\right)$ have no common predicate symbol. We use $\uparrow i \uparrow$ for the repetition of $i$-times expansions.

Proposition 2.16. A set $S$ of clauses is unsatisfiable if and only if $S^{\uparrow}$ is unsatisfiable. Furthermore if $\operatorname{rank}(S) \neq 0$, then $\operatorname{rank}(S)=\operatorname{rank}\left(S^{\uparrow}\right)+1$.

Proof. If $\operatorname{rank}(S)=0$, then $S$ includes a propositional contradictory instance. The operation of expansion does not affect this instance. If $\operatorname{rank}(S)>0$, then there is a contradictory instance $S^{\prime}$ of $S$. For $S^{\prime}$ the operation of expansion clearly keeps the property of nominal contradiction, and decreases the rank by one. The converse is also obvious because we can easily recover the contradictory instance of $S$ from the contradictory instance of $S^{\uparrow}$.

Definition 2.17. Let $\mathbb{L}_{P}(S)=\left\{P_{1}, \cdots, P_{n}\right\}$, and $\underline{x}_{i}$ be a list of new distinct variables where the length of $\underline{x}_{i}$ is the arity of $P_{\imath}$. Let $R$ be a mapping from $\left\{P_{1}\left(\underline{x}_{1}\right), \cdots, P_{n}\left(\underline{x}_{n}\right)\right\}$ to the set of all sets of clauses where predicates are taken from $\mathbb{L}_{P}\left(S^{\prime}\right), R$ is said to be a transformation from $L_{P}(S)$ to $\mathbb{L}_{P}\left(S^{\prime}\right)$ if no other variable except the variables in $\underline{x}_{i}$ occurs in the set $P_{\imath}\left(\underline{x}_{i}\right)^{R}$ of clauses which is obtained from $P_{\imath}\left(\underline{x}_{2}\right)$ by mapping $R$. The set of all transformations is denoted by $\left(\boldsymbol{L}_{P}(S) \rightarrow \boldsymbol{L}_{P}\left(S^{\prime}\right)\right)$. A transformation $R \in\left(\boldsymbol{L}_{P}(S) \rightarrow \boldsymbol{L}_{P}\left(S^{\prime}\right)\right)$ is said to be flat if no other term except the variables in $\underline{x}_{i}$ occurs in $P_{2}\left(\underline{x}_{i}\right)^{R}$. The set of all flat transformations is denoted by $\left[\boldsymbol{L}_{\boldsymbol{P}}(S) \rightarrow \boldsymbol{L}_{P}\left(S^{\prime}\right)\right] . \quad P_{\imath}\left(\underline{x}_{i}\right)^{R}$ is allowed to be taken either true, i.e. $\phi$, or false, i.e. $\{\square\}$, where $\square$ is the empty clause.

In the sequel $\boldsymbol{L}_{P}(R)$ denotes the set of all predicates that appears in $\left\{P_{2}\left(\underline{x}_{2}\right)^{R} \mid P_{\imath} \in L_{P}(S)\right\}$. It is noted that an atom $P\left(t_{1}, \cdots, t_{n}\right)$ is rewritten as $P(\underline{x})^{\left.i t_{1} / x_{1}, \cdots, t_{n} / x_{n}\right\}}$.

Dewinition 2.18. Let $R$ be an element of $\left(\boldsymbol{L}_{P}(S) \rightarrow \boldsymbol{L}_{P}\left(S^{\prime}\right)\right)$. For an atom $P\left(t_{1}, \cdots, t_{n}\right)$ where $P \subseteq \boldsymbol{L}_{P}(S)$, we define the transformation by $R$ with $P\left(t_{1}, \cdots t_{n}\right)^{R}$ $=P\left(x_{1}, \cdots, x_{n}\right)^{R\left[t_{1} / x_{1}, \cdots t_{n} / x_{n}\right]}$. For the negation of atom, we set $\left(\neg P_{2}\left(t_{1}, \cdots, t_{n}\right)\right)^{R}$ $=\left(\sim P\left(x_{1}, \cdots, x_{n}\right)^{R}\right)^{\left\{t_{1} / x_{1}, \cdots, t_{n} / x_{n}\right\}}$ where $\sim P\left(x_{1}, \cdots, x_{n}\right)^{R}$ is the conjunctive normal form of $\neg\left(P\left(x_{1}, \cdots, x_{n}\right)^{R}\right) . \neg P\left(x_{1}, \cdots, x_{n}\right)^{R}$ is $\{\square\}$ if $P\left(x_{1}, \cdots, x_{n}\right)^{R}=\phi$, and $\neg P\left(x_{1}, \cdots, x_{n}\right)^{R}$ is $\phi$ if $P\left(x_{1}, \cdots, x_{n}\right)^{R}=\{\square\}$. For a clause $C=\left\{L_{1}, \cdots, L_{n}\right\}$ where $L_{\imath}{ }^{R}=\left\{C_{i, \jmath} \mid j=1, \cdots, k_{\imath}\right\}, C^{R}$ is $\left\{\bigcup_{i=1}{ }^{n} C_{i, \jmath(\imath)} \mid C_{i, \jmath(\imath)} \in L_{\imath}{ }^{R}\right\}$. For a set $S$ of clauses, $S^{R}$ is $\cup\left\{C^{R} \mid C \in S\right\}$. 
Proposition 2.19. The set $\left[L_{P}(S) \rightarrow L_{P}\left(S^{\prime}\right)\right]$ is finite。

Proof. The number of atoms in $P\left(x_{1}, \cdots, x_{n}\right)^{R}$ is finite by the condition of flatness, and consequently the possible number of literals, clauses, and sets of clauses are finite. This means that the range of $R$ is finite.

Proposition 2.20. If a set $S$ of clauses is unsatisfiable, then $S^{R}$ is also unsatisfiable and $\operatorname{rank}\left(S^{R}\right) \leqq \operatorname{rank}(S)$.

We postpone the proof of this proposition after Lemma 4.2.

\section{§3. An Unsatisfiability-satisfiability Prover}

We define an unsatisfiability-satisfiability prover in this section, and show its soundness.

Definition 3.1. A set $S^{\prime}$ of clauses is a quasi-contraction of a set $S$ of clauses if and only if for any clause $C$ in $S$ there exists a clause $C^{\prime}$ in $S^{\prime}$ and $C^{\prime} \subset C$. This relation is denoted by $S^{\prime} \angle S$.

It is noted that $S \angle S$, and this is said to be an identity quasi-contraction. If $S^{\prime} \angle S$ then $S^{\prime} \ll S$. Therefore the following proposition is obvious from Proposition 2.12 .

Proposition 3.2. If $S^{\prime} \angle S$, and $S$ is unsatisfiable, then $S^{\prime}$ is also unsatisfiable and $\operatorname{rank}\left(S^{\prime}\right) \leqq \operatorname{rank}(S)$.

Definition 3.3. A sequence of sets of clauses $\left\langle S_{1}, \cdots, S_{2 N+1}\right\rangle$ is said to be a configuration of $S$ if and only if all the following conditions (1)-(4) are satisfied;

(1) $S_{1}=S$,

(2) $S_{2(i+1)}=S_{2 i+1}{ }^{\uparrow}, S_{2 i+1} \angle S_{2 i}$ for any $0 \leqq i \leqq N$,

(3) $S_{i}$ does not contain a propositional contradictory set of clauses for any $i<2 N+1$.

(4) and there exists no $R \in\left[\boldsymbol{L}_{\boldsymbol{P}}\left(S_{2 j+1}\right) \rightarrow \boldsymbol{L}_{\boldsymbol{P}}\left(S_{2 i+1}\right)\right]$ such that $S_{2 j+1}{ }^{R} \gg S_{2 i+1}$ for any $i$ and $j$ such that $i<j<N$.

Proposition 3.4. Let $\left\langle S_{1}, \cdots, S_{2 N+1}\right\rangle$ be a configuration, if $S_{j}$ is satisfiable then for any $i<j, S_{i}$ is satisfiable.

Proof. This is obvious from Propositions 2.16 and 3.2.

Definition 3.5. Let $\left\langle S_{1}, \cdots, S_{2 N+1}\right\rangle$ be a configuration such that every quasicontraction is an identity one, and $S_{2 N+1}$ contains a propositional contradictory set of clauses, then this is said to be the contradictory configuration. 
It is noted that the contradictory configuration of $S$ is unique.

The following proposition is obvious from Proposition 2.16.

Proposition 3.6. For a set $S$ of clauses, $\operatorname{rank}(S)=N$ if and only if $S$ has the contradictory configuration with length $2 N+1$.

Definitiom 3.7. Let $\left\langle S_{1}, \cdots, S_{2 N+1}\right\rangle$ be a configuration. For some $K<N$ there exists an $R \in\left[\boldsymbol{L}_{P}\left(S_{2 N+1}\right) \rightarrow \boldsymbol{L}_{P}\left(S_{2 K+1}\right)\right]$ such that $S_{2 N+1}{ }^{R} \gg S_{2 K+1}$ then the configuration is said to be a simple satisfactory configuration and $S_{2 K+1}$ is said to be a base set.

It is noted that if $\left\langle S_{1}, \cdots, S_{2 N+1}\right\rangle$ is a simple satisfactory configuration of which $S_{2 K+1}$ is the base set, then the configuration which have the identity quasi-contraction from $S_{2 m-1}$ to $S_{2 m}$ for $m>K$, is also a simple satisfactory configuration.

Proposition 3.8. If $S$ has a simple satisfactory configuration, then $S$ is satisfiable,

Proof. By the definition there is an $R \in\left[\boldsymbol{L}_{\boldsymbol{P}}\left(S_{2 N+1}\right) \rightarrow \boldsymbol{L}_{\boldsymbol{P}}\left(S_{2 K+1}\right)\right]$ such that $S_{2 N+1}{ }^{R} \gg S_{2 K+1}$. If $S_{2 K+1}$ is unsatisfiable then $\operatorname{rank}\left(S_{2 K+1}\right) \geqq N-K$, and by Propositions 2.16 and 3.2, $\operatorname{rank}\left(S_{2 N+1}\right)<\operatorname{rank}\left(S_{2 K+1}\right)$, and by Proposition 2.20, $\operatorname{rank}\left(S_{2 K+1}\right) \leqq \operatorname{rank}\left(S_{2 N+1}\right)$. Because this is a contradiction, $S_{2 K+1}$ is satisfiable, and also $S$ is satisfiable by Proposition 3.4.

We give a proof procedure which returns the value SAT or UNSAT for the input singleton sequence $\langle S\rangle$ of a set of clauses. It is noted that we treat the clausal form of negation of a formula, and therefore if the value is UNSAT, then the original formula is a theorem of the first order logic, and if the value is SAT, then that is not a theorem.

Function test (Seq: a sequence of sets of clauses): (SAT, UNSAT);

Let Seq be $\left\langle S_{1}, \cdots, S_{2 N+1}\right\rangle$.

Step 1: If the propositional variable part of $S_{2 N+1}$ is unsatisfiable then return (UNSAT);

Step 2: If for some $K<N$ there is some $R \in\left[\boldsymbol{L}_{P}\left(S_{2 N+1}\right) \rightarrow \boldsymbol{L}_{P}\left(S_{2 K+1}\right)\right]$ such that $S_{2 N+1}{ }^{R} \gg S_{2 K+1}$, then return (SAT);

Let $\left\{S_{2 N+1}{ }^{\uparrow} 1, \cdots, S_{2 N+1}{ }^{\uparrow} n\right.$ be the set of all quasi-contractions of $S_{2 N+1}{ }^{\uparrow}$, and evaluate test $\left(\left\langle S_{1}, \cdots, S_{2 N+1}, S_{2 N+1}{ }^{\uparrow}, S_{2 N+1}{ }^{\uparrow}{ }_{1}\right\rangle\right), \cdots$, test $\left(\left\langle S_{1}, \cdots, S_{2 N+1}, S_{2 N+1}{ }^{\uparrow}, S_{2 N+1}{ }^{\uparrow}\right\rangle\right)$ in \parallel ;

Step 3: If test $\left(\left\langle S_{1}, \cdots, S_{2 N+1}, S_{2 N+1}{ }^{\uparrow}, S_{2 N+1}{ }^{\uparrow}\right\rangle\right)$ takes the value UNSAT then return(UNSAT), else if for some $i$ test $\left(\left\langle S_{1}, \cdots, S_{2 N+1}, S_{2 N+1}{ }^{\uparrow}, S_{2 N+1}{ }^{\uparrow} i\right\rangle\right)$ takes the value $\mathrm{SAT}$ then return $(\mathrm{SAT})$;

end. 
It is noted that at Step 2 the simple subsumption check is effectively computable, and therefore Step 2 is effectively computable, but not efficient.

Proposition 3.9. During the evaluation of test $(\langle S\rangle)$, if test $(S e q)$ is called recursively then $S e q$ is a configuration of $S$.

Proof. We use the induction on the number of recursive calls of test. For the first call, $\langle S\rangle$ is a configuration of $S$. If test $\left(\left\langle S_{1}, \cdots, S_{2 N+1}, S_{2 N+1}{ }^{\uparrow}, S_{2 N+1}{ }^{\uparrow} i\right\rangle\right)$ is called in test $\left(\left\langle S_{1}, \cdots S_{2 N+1}\right\rangle\right)$, then obviously $\left\langle S_{1}, \cdots, S_{2 N+1}, S_{2 N+1}{ }^{\uparrow}, S_{2 N+1}{ }^{\uparrow}\right\rangle$ is a configuration of $S$ from the contents of the function test, and by the induction hypothesis.

Theorem 3.10. The value of test $(\langle S\rangle)$ is UNSAT if and only if $S$ has the contradictory configuration.

Proof. First we show that if test $\left(\left\langle S_{1}, \cdots, S_{2 N+1}\right\rangle\right)$ takes the value UNSAT, then $S_{2 N+1}$ has a contradictory configuration. We use the induction on the number of recursive calls of test. The procedure returns with the value UNSAT in two cases. In the first case that $S_{2 N+1}$ includes a propositionally unsatisfiable subsets, $S_{2 N+1}$ has obviously a contradictory configuration. In the second case that test $\left(\left\langle S_{1}, \cdots, S_{2 N+1}, S_{2 N+1}{ }^{\uparrow}, S_{2 N+1}{ }^{\uparrow}\right\rangle\right)$ takes the value UNSAT, $S_{2 N+1}{ }^{\uparrow}$ has the contradictory configuration $\left\langle S_{2 N+1}{ }^{\uparrow}, \cdots, S_{2 M+1}\right\rangle$ by the induction hypothesis, and hence $\left\langle S_{2 N+1}, S_{2 N+1}{ }^{\uparrow}, S_{2 N+1}{ }^{\uparrow}, \cdots, S_{2 M+1}\right\rangle$ is obviously a contradictory configuration of $S_{2 N+1}$.

Next we show the converse. Let $\left\langle S_{1}, \cdots, S_{2 N+1}\right\rangle$ be the contradictory configuration, we show that test $\left(\left\langle S_{1}, \cdots, S_{2(N-m)+1}\right\rangle\right)$ takes the value UNSAT by the induction on $m$. If $m=0$, then $S_{2 N+1}$ includes a propositionally unsatisfiable set of clauses, so that the procedure obviously returns the value UNSAT on the step 1. Let $m=k+1$, then test $\left(\left\langle S_{1}, \cdots, S_{2(N-k-1)+1}, S_{2(N-k-1)+1}{ }^{\uparrow}, S_{2(N-k)+1}\right\rangle\right)$ where $S_{2(N-k-1)+1}{ }^{\uparrow}=S_{2(N-k)+1}$, takes the value UNSAT by the induction hypothesis, and therefore test $\left(\left\langle S_{1}, \cdots, S_{2(N-k-1)+1}\right\rangle\right)$ obviously takes the value UNSAT.

Theorem 3.11. The value of test $(\langle S\rangle)$ is $S A T$ if and only if $S$ has a simple satisfactory configuration.

Proof. First we show that if $S$ has a simple satisfactory configuration, then the value of test $(\langle S\rangle)$ is SAT. Let $\left\langle S_{1}, \cdots, S_{2 N+1}\right\rangle$ be a simple satisfactory configuration of $S$. By the assumption $S$ is satisfiable, so that test $\left(\left\langle S_{1}, \cdots, S_{2 \imath+1}\right\rangle\right)$ does not return the value UNSAT for any $i \leqq N$. We show that test $\left(\left\langle S_{1}, \cdots\right.\right.$, $\left.\left.S_{2(N-m)+1}\right\rangle\right)$ takes the value SAT by the induction on $m$. If $m=0$, this is obvious from the definition. Let $m=k+1$, then test $\left(\left\langle S_{1}, \cdots, S_{2(N-k-1)+1}, S_{2(N-k-1)+1}{ }^{\uparrow}\right.\right.$, $\left.\left.S_{2(N-k)+1}\right\rangle\right)$, where $S_{2(N-k)+1} \angle S_{2(N-k-1)+1}{ }^{\uparrow}$, takes the value SAT by the induction 
hypothesis and obviously test $\left(\left\langle S_{1}, \cdots, S_{2(N-k-1)+1}\right\rangle\right)$ takes the value SAT on the step 3.

Next we show the converse. The procedure test returns with the value SAT in Step 2 or 3, but it is obvious that Step 2 is essential. By Proposition $3.9, S$ has obviously a simple satisfactory configuration.

Theorem 3.10 means the completeness of the procedure test in the sense of the ordinary theorem proving method.

Definition 3.12. Let $\left\langle S_{1}, \cdots, S_{2 N+1}\right\rangle$ be a configuration. If for some $K<N$ there exists an $R \in\left[\boldsymbol{L}_{\boldsymbol{P}}\left(S_{2 N+1}\right) \rightarrow \boldsymbol{L}_{\boldsymbol{P}}\left(S_{2 K+1}\right)\right]$ and there exists a set $M$ of clauses such that it includes $S_{2 K+1}$ and $M^{\uparrow N-K \uparrow R} \gg M$, and for any $i \leqq N-K, M^{\uparrow i \uparrow}$ does not include a propositional contradictory instance, then the configuration is said to be an elementary satisfactory configuration by $R$ and $M$, and $M$ is said to be a saturated set for $S_{2 K+1}$ and $R$.

Proposition 3.13. If $S$ has an elementary satisfactory configuration, then $S$ is satisfiable.

Proof. By the similar argument to Proposition 3.8, $M$ is satisfiable. Because $M$ includes $S_{2 K+1}, S_{2 K+1}$ is satisfiable. Consequently $S$ is satisfiable from Proposition 3.4 .

It is noted that a simple satisfactory configuration is an elementary satisfactory configuration. The following is a modification of a function test to check the elementary satisfactory configuration of $S$.

Function test 2(Seq: a sequence of sets of clauses): (SAT, UNSAT);

Let Seq be $\left\langle S_{1}, \cdots, S_{2 N+1}\right\rangle$.

Step 1: If the propositional variable part of $S_{2 N+1}$ is unsatisfiable then return (UNSAT);

Evaluate Step 2 and 3 in parallel.

Step 2: For every $K<N$ and every $R \in\left[\boldsymbol{L}_{P}\left(S_{2 N+1}\right) \rightarrow \boldsymbol{L}_{P}\left(S_{2 K+1}\right)\right]$, evaluate $Q_{1}\left(S_{2 K+1}, S_{2 N+1}{ }^{R}, R, N-K\right)$ in parallel. If one of them takes the value SAT then return (SAT);

Let $\left\{S_{2 N+1}{ }^{\uparrow}{ }_{1}, \cdots, S_{2 N+1}{ }^{\uparrow}{ }_{n}\right\}$ be the set of all quasi-contractions of $S_{2 N+1}{ }^{\uparrow}$, and evaluate test $2\left(\left\langle S_{1}, \cdots, S_{2 N+1}, S_{2 N+1}{ }^{\uparrow}, S_{2 N+1}{ }^{\uparrow}{ }_{1}\right\rangle\right), \cdots$, test $2\left(\left\langle S_{1}, \cdots, S_{2 N+1}, S_{2 N+1}{ }^{\uparrow}\right.\right.$, $\left.\left.S_{2 N+1}{ }^{\uparrow}{ }_{n}\right\rangle\right)$ in parallel ;

Step 3: If test $2\left(\left\langle S_{1}, \cdots, S_{2 N+1}, S_{2 N+1}{ }^{\uparrow}, S_{2 N+0}{ }^{\uparrow}\right\rangle\right)$ takes the value UNSAT then return(UNSAT), else if test $\left(\left\langle S_{1}, \cdots, S_{2 N+1}, S_{2 N+1}{ }^{\uparrow}, S_{2 N+1}{ }^{\uparrow}\right\rangle\right)$ takes the value SAT for some $i$ then return (SAT);

end ;

Function $Q_{1}\left(S, S^{\prime}:\right.$ sets of clauses, $R:$ a flat transformation, $m:$ integer): (SAT, 
UNSAT);

If $S^{\prime} \gg S$, then return $(\mathrm{SAT})$;

Let $\left\{S^{\prime}{ }_{1}, \cdots, S_{n}{ }_{n}\right\}$ be the set of all quasi-contractions of $S^{\prime}$, and evaluate $Q_{2}\left(S \cup S^{\prime}{ }_{1}, R, m\right), \cdots, Q_{2}\left(S \cup S^{\prime}{ }_{n}, R, m\right)$ in parallel ;

If $Q_{2}\left(S \cup S^{\prime}, R, m\right)$ takes the value UNSAT then return(UNSAT), else if $Q_{2}\left(S \cup S^{\prime}{ }_{i}, R, m\right)$ takes the value SAT for some $i$ then return(SAT);

end ;

Function $Q_{2}(S:$ a set of clauses, $R:$ a flat transformation, $m:$ integer): (SAT, UNSAT);

Variable $S^{\prime}$ : a set of clauses, $i$ : integer ;

$S^{\prime}:=S ; i:=0$;

While $i<m$ do $S^{\prime}=S^{\uparrow}$;

If the propositional variable part of $S^{\prime}$ is unsatisfiable then return(UNSAT); $\operatorname{return}\left(Q_{1}\left(S, S^{\prime}, R, m\right)\right)$;

end.

Proposition 3.14. The value of test $2(\langle S\rangle)$ is UNSAT if and only if $S$ has the contradictory configuration.

We omit the proof of Proposition 3.14 because this is almost the same as the proof of Theorem 3.10 for the function test.

Proposition 3.15. If the value of test $2(\langle S\rangle)$ is $S A T$, then $S$ has an elementary satisfactory configuration.

Sketch of proof. We show that if the value of $Q_{1}\left(S, S^{\prime}, R, K\right)$ is SAT, where $S^{\prime}$ is a quasi-contraction of $S^{\uparrow{ }^{\uparrow}}$ which does not include a propositional contradictory instance, then there is an elementary satisfactory sequence by $R$ and some $M$ such that $S \subset M$. We show this by the induction on the number of recursive calls of $Q_{1}$. The first case is that $S^{\prime R} \gg S$ holds. In this case we can obviously take $S$ for a saturated set for $S$ and $R$. The other case is that the value of $Q_{2}\left(S \cup S^{\prime \prime}, R, K\right)$ is SAT for some quasi-contraction $S^{\prime \prime}$ of $S^{\prime R}$. If the value of $Q_{2}\left(S \cup S^{\prime \prime}, R, K\right)$ is SAT, then the value of $Q_{1}\left(S \cup S^{\prime \prime},\left(S \cup S^{\prime \prime}\right)^{R}, R, K\right)$ is SAT where $\left(S \cup S^{\prime \prime}\right)^{R}$ does not include propositional contradictory instance. Hence by the induction hypothesis, there is an elementary satisfactory sequence by $R$ and some $M$ such that $S \subset S \cup S^{\prime \prime} \subset M$.

Proposition 3.16. If $S$ has an elementary satisfactory configuration, then the value of test $2(\langle S\rangle)$ is $S A T$.

Sketch of proof. Let $\left\langle S_{1}, \cdots, S_{2 N+1}\right\rangle$ be an elementary satisfactory configuration by $R \in\left[\boldsymbol{L}_{\boldsymbol{P}}\left(S_{2 N+1}\right) \rightarrow \boldsymbol{L}_{P}\left(S_{2 K+1}\right)\right]$ and $M$ which includes $S_{2 K+1}$. It is enough to show that the value of $Q_{1}\left(S_{2 K+1}, S_{2 K+1}{ }^{\uparrow-K \uparrow R}, R, N-K\right)$ is SAT. 
$A$ set $S^{\prime}$ of clauses is said to be strictly simply subsumed by $M$ if for any clause $C$ in $S^{\prime}$ which is not a tautology, there exist a clause $C^{\prime}$ in $M$ and a substitution $\theta$ such that $C=C^{\prime \theta}$, and this relation is denoted by $S^{\prime} \stackrel{\longrightarrow}{\rightarrow} M$. We show that the value of $Q_{1}\left(S^{\prime}, S^{\prime \uparrow N-K \uparrow R}, R, N-K\right)$ where $S \subset S^{\prime}$ and $S^{\prime} \cong M$, is SAT. If $S^{\prime \uparrow N-K \uparrow R} \gg S^{\prime}$, then there is nothing to prove. Otherwise there is a clause $C \in S^{\prime \uparrow N-K \uparrow R}$ which is not simply subsumed by $S^{\prime}$. By the assumption $S^{\prime \uparrow N-K \uparrow R} \subset M^{\uparrow N-K \uparrow R} \gg M$, we can take a quasi-contraction $C^{\prime}$ of $C$ such that $C^{\prime}=C^{\prime \prime}{ }^{\theta}$ for some $C^{\prime \prime} \in M$. Collecting these and tautologies, we can take $S^{\prime \prime}$ such that $S^{\prime \prime}$ is a quasi-contraction of $S^{\prime \uparrow N-K \uparrow R}$ and $S^{\prime \prime} \rightarrow M$. The evaluation of $Q_{2}\left(S^{\prime} \cup S^{\prime \prime}, R, N-K\right)$ leads to the evaluation of $Q_{1}\left(S^{\prime} \cup S^{\prime \prime}, S^{\prime} \cup S^{\prime \prime \uparrow N-K \uparrow R}, R, N-K\right)$ by the assumption for $M$. Hence the fact that the height of set of clauses does not increase after either a expansion or a flat transformation, and $\left\{S^{\prime} \mid S^{\prime} \underset{\rightarrow}{\rightarrow} M\right.$ and height $\left.\left(S^{\prime}\right)<L\right\}$ is finite for any $L$ except the choice of variables and ignorance of tautologies, implies Proposition 3.16.

\section{§4. Elementary Properties}

In this section we show some propositions related to the satisfiability. In $\S 5$.we show an application of results in this section.

Proposition 4.1. Let $C$ be a clause, $\theta$ be a substitution for $C$, and $\varepsilon$ be $a$ decomposition of $C^{\theta}$, then there exist some decomposition $\varepsilon^{\prime}$ of $C$, and a substitution $\theta^{\prime}$ for $C^{\varepsilon^{\prime}}$ such that $C^{\theta \varepsilon}=C^{\varepsilon^{\prime} \theta^{\prime}}$ holds.

Proof. Let $x$ be any variable occurring in the terms to be substituted by $\theta$. We consider three cases on $x^{\theta}$.

(1) If $x^{\theta}=c$ for a constant $c$, then we set $x^{s^{\prime}}=c$.

(2) If $x^{\theta}=y$ for a variable, we consider two cases on $y^{\varepsilon}$ from the definition of the decomposition.

(2.1) If $y^{\varepsilon}=c$ for a constant $c$ then we set $x^{\varepsilon^{\prime}}=c$.

(2.2) If $y^{\varepsilon}=f\left(u_{1}, y, \cdots, u_{n, y}\right)$ for a function $f$ and for a new distinct sequence $\left\langle u_{1, y}, \cdots, u_{n, y}\right\rangle$ of variables, then we set $x^{\varepsilon^{\prime}}=f^{\prime}\left(u_{1, x}, \cdots, u_{n, x}\right)$, and $u_{\imath, x}{ }^{{ }^{\prime}}$ $=u_{i, y}$ for any $i$.

(3) If $x^{\theta}=f\left(t_{1}, \cdots, t_{n}\right)$ for a function $f$, then we set $x^{\varepsilon^{\prime}}=f\left(u_{1, x}, \cdots, u_{n, x}\right)$, $u_{i, x^{\theta^{\prime}}}=t_{i}^{\varepsilon}$ for any $i$.

For example, let $C$ be $P(x, y) \vee Q(z, f(y, u))$ and $\theta$ be $\left\{c / x, v / y, w / z, f\left(c, x_{1}\right) / u\right\}$, and $\varepsilon$ be $\left\{c / v, f\left(w_{1}, w_{2}\right) / w, f\left(x_{2}, x_{3}\right) / x_{1}\right\}$, then $C^{\theta}$ is $P(c, v) \vee Q\left(w, f\left(v, f\left(c, x_{1}\right)\right)\right)$ and $C^{\theta \varepsilon}$ is $P(c, c) \vee Q\left(f\left(w_{1}, w_{2}\right), f\left(c, f\left(c, f\left(x_{2}, x_{3}\right)\right)\right)\right)$. Let $\varepsilon^{\prime}$ be $\left\{c / x, c / y, f\left(z_{1}, z_{2}\right) / z\right.$, $\left.f\left(u_{1}, u_{2}\right) / u\right\}$ and $\theta^{\prime}$ be $\left\{w_{1} / z_{1}, w_{2} / z_{2}, c / u_{1}, f\left(x_{2}, x_{3}\right) / u_{2}\right\}$, then $C^{\varepsilon^{\prime}}$ is $P(c, c) \vee$ $Q\left(f\left(z_{1}, z_{2}\right), f\left(c, f\left(u_{1}, u_{2}\right)\right)\right)$ and $C^{\theta \varepsilon}=C^{\varepsilon^{\prime} \theta^{\prime}}$ holds obviously.

It is noted that we can easily see in the proof of Proposition 4.1 that the height of $\theta^{\prime}$ is less than or equal to the height of $\theta$. 
Lemma 4.2. If a set $S$ of clauses is a nominal contradiction, then $S^{R}$ is a nominal contradiction.

Proof. By the assumption, $S$ is false under any assignment of truth values for literal atoms in $S$. Then if an assignment $\alpha$ of truth values for literal atoms in $S^{R}$ is given, then we can set the assignment $\beta$ of truth values for atoms in $S$ by $\beta\left(P\left(t_{1}, \cdots, t_{n}\right)\right)=V\left(\alpha, P\left(t_{1}, \cdots, t_{n}\right)^{R}\right)$ where $V$ denotes the valuation of truth values. Under these assignment we can show that $V(\beta, \neg A)=$ $V\left(\alpha, \sim A^{R}\right)$, and $V(\beta, C)=V\left(\alpha, C^{R}\right)$. Because $V(\beta, S)$ is false for any $\beta, V\left(\alpha, S^{R}\right)$ is also false for any $\alpha$. This implies Lemma 4.2 .

For example, let $S$ be $\{\{P(x)\},\{\neg P(x)\}\}$ and $P(x)^{R}$ be $Q(x) \vee R(x)$, then $S^{R}$ is $\{\{Q(x), R(x)\},\{\neg Q(x)\},\{\neg R(x)\}\}$.

Proposition 4.3. Let $\theta$ be a substitution for a clause $C$, then $C^{\theta R}=C^{R \theta}$.

Proof. Let $\pi$ be $\left\{t_{1} / x_{1}, \cdots, t_{k} / x_{k}\right\}$. For an atom $P\left(t_{1}, \cdots, t_{k}\right)$, we have $P\left(t_{1}, \cdots, t_{k}\right)^{\theta R}=P\left(t_{1}{ }^{\theta}, \cdots, t_{k}{ }^{\theta}\right)^{R}=P\left(x_{1}, \cdots, x_{k}\right)^{R \pi \theta}=P\left(t_{1}, \cdots, t_{k}\right)^{R \theta}$. For a negation of atom, $\neg P\left(t_{1}, \cdots, t_{k}\right)$ we have, $\left(\neg P\left(t_{1}, \cdots, t_{k}\right)\right)^{\theta R}=\left(\neg P\left(t_{1}{ }^{\theta}, \cdots, t_{k}{ }^{\theta}\right)\right)^{R}=$ $\left(\sim P\left(x_{1}, \cdots, x_{k}\right)^{R}\right)^{\pi \theta}=\left(\neg P\left(t_{1}, \cdots, t_{k}\right)\right)^{R \theta}$. Hence for a clause $C=\left\{L_{1}, \cdots, L_{n}\right\}$, we have $C^{\theta R}=\left\{\cup\left\{C_{i} \mid C_{i} \in L_{2}{ }^{\theta R}\right\}\right\}=\left\{\cup\left\{C_{i} \mid C_{i} \in L_{i}{ }^{R \theta}\right\}\right\}=C^{R \theta}$.

Proof of Proposition 2.20. By the assumption there are clauses $C_{1}, \cdots, C_{n} \in S$, and substitutions $\theta_{1}, \cdots, \theta_{n}$ such that $\left\{C_{1}{ }^{\theta_{1}}, \cdots, C_{n}{ }^{\theta_{n}}\right\}$ is a nominal contradiction. Hence by Lemma 4.2, $\left\{C_{1}{ }^{\theta_{1}}, \cdots, C_{n}{ }^{\theta_{n}}\right\}^{R}$ is a nominal contradiction. By Proposition 4.3, this can be rewritten $\left\{C_{1}{ }^{R \theta_{1}}, \cdots, C_{n}{ }^{R \theta_{n}}\right\}$. Therefore $S^{R}$ is unsatisfiable, and $\operatorname{rank}\left(S^{R}\right) \leqq \operatorname{rank}(S)$.

Roughly speaking, the relation that a clause $C$ is subsumed by some $n$ clauses in $S$ is kept through operations of quasi-contractions and expansions but perhaps not through flat transformations. Therefore we introduce the next definition of a relation, and Propositions 4.5, 4.6 and Theorem 4.7 show that the relation is kept through each operation.

Definition 4.4. A clause $C$ is pseudo-subsumed by clauses $C_{1}, \cdots, C_{n}$ in a set $S$ of clauses if there exist some $n$ substitutions $\theta_{1}, \cdots, \theta_{n}$, and some clauses $D_{1}, \cdots, D_{m}$ in $S$, and substitutions $\pi_{1}, \cdots, \pi_{m}$ such that there hold three conditions that $\left\{\psi\left(D_{1}{ }^{\pi_{1}}, C\right\}, \cdots, \psi\left(D_{m}{ }^{\pi} m, C\right)\right\}$ is a nominal contradiction, $C \subset \cup_{i=1}{ }^{n} C_{i}{ }^{\theta_{i}}$, and $\left\{\left\langle C_{i}, \theta_{i}\right\rangle \mid i=1, \cdots, n\right\} \subset\left\{\left\langle D_{i}, \pi_{i}\right\rangle \mid i=1, \cdots, m\right\}$.

For example $P(x) \supset P(f(f(f(x))))$ is ipseudo-subsumed by $C_{1}=P(x) \supset P(f(x))$ and $C_{2}=P(x) \supset P(f(x))$ where $\theta_{1}=\pi_{1}=\{x / x\}, \quad \theta_{2}=\pi_{2}=\{f(f(x)) / x\}, \quad C_{3}=P(x) \supset$ $P(f(x))$ and $\pi_{3}=\{f(x) / x\}$. 
Proposition 4.5. Let $S^{\prime} \angle S$, and a clause $C$ be pseudo-subsumed by clauses $C_{1}, \cdots, C_{n}$ in $S$, then there exist some $C^{\prime} \subset C$ and some clauses $C_{1}{ }^{\prime}, \cdots, C_{n}{ }^{\prime}$ in $S^{\prime}$ and $C^{\prime}$ is pseudo-subsumed by clauses $C_{1}{ }^{\prime}, \cdots C_{n}{ }^{\prime}$ in $S^{\prime}$.

Proof. By the assumption there exist some $n$ substitutions $\theta_{1}, \cdots, \theta_{n}$, and some clauses $D_{1}, \cdots, D_{m}$ in $S$, and substitutions $\pi_{1}, \cdots, \pi_{m}$, and there hold the three conditions. We take $C_{1}{ }^{\prime}, \cdots, C_{n}{ }^{\prime}$, and $D_{1}{ }^{\prime}, \cdots, D_{m}{ }^{\prime}$ as the result of a quasicontraction of $C_{1}, \cdots, C_{n}$, and $D_{1}, \cdots, D_{m}$ respectively. Let $C^{\prime}$ be $C \cap \bigcup_{i=1}^{m} D_{\imath}{ }^{\prime \pi}{ }^{2}$. The conditions $C^{\prime} \subset \bigcup_{i=1}{ }^{n} C_{i}{ }^{\prime}{ }^{\theta_{i}}$, and $\left\{\left\langle C_{i}{ }^{\prime}, \theta_{i}\right\rangle \mid i=1, \cdots, n\right\} \subset\left\{\left\langle D_{i}{ }^{\prime}, \pi_{\imath}\right\rangle \mid i=1, \cdots, m\right\}$ hold obviously. If $L \in \psi\left(D_{\imath}{ }^{\prime \pi_{i}}, C^{\prime}\right)$ then obviously $L \in D_{i}{ }^{\pi_{i}}$ and $L \notin C^{\prime}$. On the other hand if $L \notin C^{\prime}$ then $L \oplus C$ or $L \notin \bigcup_{i=1}{ }^{m} D_{\imath}{ }^{\prime \pi} i$. But the formula $L \notin \bigcup_{\imath=1}{ }^{m} D_{\imath}{ }^{\prime \pi_{i}}$ contradict $L \in D_{i}{ }^{\pi} i$. Therefore $L \oplus C$ and this implies that if $L \in \phi\left(D_{i}{ }^{\prime \pi i}, C^{\prime}\right)$ then $L \in \psi\left(D_{2}{ }^{\pi_{i}}, C\right)$. That is $\left\{\psi\left(D_{1}{ }^{\prime \pi_{1}}, C^{\prime}\right), \cdots, \phi\left(D_{m}{ }^{\prime \pi_{m}}, C^{\prime}\right)\right\}$ is a quasi-contraction of $\left\{\psi\left(D_{1}^{\pi_{1}}, C\right), \cdots, \phi\left(D_{m}{ }^{\pi_{m}}, C\right)\right\}$. Hence $\left\{\psi\left(D_{1}{ }^{\pi_{1}}, C^{\prime}\right), \cdots, \phi\left(D_{m}{ }^{\prime \pi_{m}}, C^{\prime}\right)\right\}$ is obviously a nominal contradiction.

Proposition 4.6. If a clause $C$ is pseudo-subsumed by clauses $C_{1}, \cdots, C_{n}$ in $S$, and a clause $C^{\prime}$ belongs to $C^{\uparrow}$, then there exist some $C_{1}{ }^{\prime} \in C_{1}{ }^{\uparrow}, \cdots, C_{n}{ }^{\prime} \in C_{n}{ }^{\uparrow}$, and $C^{\prime}$ is pseudo-subsumed by clauses $C_{1}{ }^{\prime}, \cdots, C_{n}{ }^{\prime}$ in $S^{\uparrow}$.

Proof. By the assumption there exist some $n$ substitutions $\theta_{1}, \cdots, \theta_{n}$, and some clauses $D_{1}, \cdots, D_{m}$ in $S$, and substitutions $\pi_{1}, \cdots, \pi_{m}$, and there hold the three conditions. From $C^{\prime} \in C^{\uparrow}$, there exists some decomposition $\varepsilon$ of $C$, and $C^{\prime}=C^{\varepsilon *}$. We extend this decomposition for $\bigcup_{i=1}{ }^{m} D_{i}{ }^{\pi_{i}}$. Let the extended decomposition be $\delta$. Then $\left\{\psi\left(D_{1}{ }^{\pi_{1}}, C\right), \cdots, \psi\left(D_{m}{ }^{\pi_{m}}, C\right)\right\}^{\delta}$ is a nominal contradiction, $C^{\delta}=C^{\varepsilon} \subset \bigcup_{i=1}^{n} C_{i}{ }^{\theta_{2} \delta}$, and $\left\{\left\langle C_{\imath}, \theta_{i}\right\rangle \mid i=1, \cdots, n\right\}^{\delta} \subset\left\{\left\langle D, \pi_{\imath}\right\rangle \mid i=1, \cdots, m\right\}^{\delta}$. By Proposition 4.1, there exist some decompositions $\delta_{\imath}{ }^{\prime}$ and $\lambda_{i}{ }^{\prime}$ such that $C_{\imath}{ }^{{ }{ }^{\delta}}=C_{\imath}{ }^{\delta_{\imath}{ }^{\prime} \theta_{\imath}{ }^{\prime}}$ and $D_{\imath}{ }^{\pi} \imath^{\delta}=D_{i}{ }^{\lambda} \imath^{\prime}{ }^{\pi} \imath^{\prime}$. Let $C_{\imath}{ }^{\prime}$ be $C_{\imath}{ }^{{ }^{\delta^{\prime}}{ }^{\prime}}$, and $D_{\imath}{ }^{\prime}$ be $D_{i}{ }^{\lambda} i^{\prime}$, then by the substitutions $\theta_{1}{ }^{\prime}, \cdots, \theta_{n}{ }^{\prime}$ and $\pi_{1}{ }^{\prime}, \cdots, \pi_{m}{ }^{\prime}$, the required three conditions obviously hold.

For example let $P(x) \supset P(f(f(f(x))))$ be pseudo-subsumed by $C_{1}=P(x) \supset P(f(x))$ and $C_{2}=P(x) \supset P(f(x)) \quad$ where $\theta_{1}=\pi_{1}=\{x / x\}, \quad \theta_{2}=\pi_{2}=\{f(f(x)) / x\}, \quad C_{3}=P(x) \supset$ $P(f(x))$ and $\pi_{3}=\{f(x) / x\}$. And let $C^{\prime}=P_{g}(y) \supset P_{f}(f(f(g(y)))) \in C^{\uparrow}$ where $C^{\prime}$ is the result of a decomposition $\{g(y) / x\}$, then $C^{\prime}$ is pseudo-subsumed by $C_{1}{ }^{\prime}=$ $P_{g}(y) \supset P_{f}(g(y)) \quad$ and $\quad C_{2}{ }^{\prime}=P_{f}(y) \supset P_{f}(f(y)) \quad$ where $\quad \theta_{1}=\pi_{1}=\{y / y\}, \quad \theta_{2}=\pi_{2}=$ $\{f(f(y)) / y\}, C_{3}=P_{f}(y) \supset P_{f}(f(y))$ and $\pi_{3}=\{f(y) / y\}$.

Theorem 4.7. If a clause $C$ is pseudo-subsumed by clauses $C_{1}, \cdots, C_{n}$ in $S$, and a clause $C^{\prime}$ belongs to $C^{R}$, then there exist some $C_{1}{ }^{\prime} \in C_{1}{ }^{R}, \cdots, C_{n}{ }^{\prime} \in C_{n}{ }^{R}$, and $C^{\prime}$ is pseudo-subsumed by clauses $C_{1}{ }^{\prime}, \cdots, C_{n}{ }^{\prime}$ in $S^{R}$.

Proof. By the assumption there exist some $n$ substitutions $\theta_{1}, \cdots, \theta_{n}$, and some clauses $D_{1}, \cdots, D_{m}$ in $S$, and substitutions $\pi_{1}, \cdots, \pi_{m}$, and there hold the 
three conditions. Let $C$ be $\left\{L_{1}, \cdots, L_{M}\right\}$, then by some $C_{L_{i}}$ in $L_{\imath}{ }^{R}, C^{\prime}$ is $\cup_{i=1}{ }^{M I} C_{L_{i}}$. If $L^{\pi_{i}}$ belongs to $C$ for $L \in C_{i}$, that is, $L^{\pi_{i}}=L_{j}$ for some $j$, then $L_{j}{ }^{R}=L^{\pi_{i} R}=L^{R \pi_{i}}$, so that there exists some clause $X_{L} \in L^{R}$ such that $C_{L_{j}}=X_{L}{ }^{{ }^{i} i}$. By the definition if $C_{i}$ is $\left\{L_{i, 1}, \cdots, L_{i, n(i)}\right\}$, then $C_{i}{ }^{R}$ is $\left\{\bigcup_{s=1}{ }^{n(i)} C_{\imath, s, \jmath(s)} \mid C_{i, s, \jmath(s)} \in L_{\imath, s}{ }^{R}\right\}$. It is noted that $\left\{\left\langle C_{\imath}, \theta_{i}\right\rangle \mid i=1, \cdots, n\right\}$ is included in $\left\{\left\langle D_{i}, \pi_{i}\right\rangle \mid i=1, \cdots, m\right\}$. In $C_{\imath}{ }^{R}$ we choose $C_{i}{ }^{\prime}=\bigcup_{s=1}{ }^{n(i)} C_{\imath, s, j(s)}$ such that $C_{i, s, j(s)}$ is the above $X_{L(\imath, s)}$ for any such that $L_{i, s}{ }^{\theta_{i}}$ belongs to $C$. It is obvious that $C^{\prime} \subset \bigcup_{i=1}{ }^{n} C_{i}{ }^{{ }^{\theta}}{ }^{i}$ because $C \subset \bigcup_{i=1}{ }^{n} C_{i}{ }^{\theta}{ }^{i}$. For $D_{\imath}$ we use a similar argument but in this case we choose a set $D_{\imath}^{\prime}$ of clauses. By the definition if $D_{i}=\left\{L_{i, 1}, \cdots, L_{i, n(\imath)}\right\}$, then $D_{i}{ }^{R}=\left\{\bigcup_{s=1}{ }^{n(i)} C_{\imath, s, j(s)} \mid C_{i, s, j(s)} \in\right.$ $\left.L_{i, s}{ }^{R}\right\}$. Hence we take a set $D_{\imath}{ }^{\prime}=\left\{\bigcup_{s=1}{ }^{n(i)} C_{i, s, \jmath(s)} \mid C_{i, s, j(s)} \in L_{i, s}{ }^{R}\right.$, and if $L_{i, s}{ }^{\pi}$ belongs to $C$, then $C_{i, s, \jmath(s)}$ is the above $\left.X_{L(i, s)}\right\}$. We show that $C^{\prime}$ is pseudosubsumed by clauses $C_{1}{ }^{\prime}, \cdots, C_{n}{ }^{\prime}$ using $\bigcup_{i=1}{ }^{m}\left\{\left\langle D, \pi_{2}\right\rangle \mid D \in D_{i}{ }^{\prime}\right\}$. For the first condition the set of clauses that we have to consider becomes $\left\{\psi\left(D^{\pi_{i}}, C^{\prime}\right) \mid D \in D_{1}{ }^{\prime}\right\}$ $\cup \cdots \cup\left\{\left(\psi\left(D^{\pi} m, C^{\prime}\right) \mid D \in D_{m}{ }^{\prime}\right\}\right.$. To show that this set is a nominal contradiction, it is enough to show that $\left\{\bigcup_{s=1}{ }^{n(1)} C_{1, s, j(s)} \mid C_{1, s, j(s)} \in L_{1, s}{ }^{R}\right.$, and if $L_{1, s}{ }^{\pi_{1}}$ belongs to $C$, then $C_{1, s, j(s)}$ is $\left.\phi\right\} \cup \cdots \cup\left\{\bigcup_{s=1}{ }^{n(m)} C_{m, s, j(s)} \mid C_{m, s, j(s)} \in L_{m, s}{ }^{R}\right.$, and if $L_{m, s}{ }^{\pi_{m}}$ belongs to $C$, then $C_{m, s, j(s)}$ is $\left.\phi\right\}$ is a nominal contradiction. This set is equal to $\psi\left(D_{i}{ }^{\pi} i, C\right)^{R} \cup \cdots \cup \psi\left(D_{m}{ }^{\pi} m, C\right)^{R}$, and is a nominal contradiction by Lemma 4.2. The last condition obviously holds.

For example let $P(x) \supset P(f(f(f(x))))$ be pseudo-subsumed by $C_{1}=P(x) \supset P(f(x))$ and $C_{2}=P(x) \supset P(f(x))$ where $\theta_{1}=\pi_{1}=\{x / x\}, \quad \theta_{2}=\pi_{2}=\{f(f(x)) / x\}, \quad C_{3}=P(x) \supset$ $P(f(x))$ and $\pi_{3}=\{f(x) / x\}$. Let $P(x)^{R}=Q_{1}(x) \wedge Q_{2}(x)$, and let $C^{\prime}$ be $Q_{1}(x) \wedge Q_{2}(x) \supset$ $Q_{1}(f(f(f(x)))) \in C^{R}$. The clause $C^{\prime}$ is the union of $\left\{\neg Q_{1}(x), \neg Q_{2}(x)\right\}$ and $\left\{Q_{1}(f(f(f(x))))\right\} \quad$ which are elements of $\neg P(x)^{R}=\left\{\left\{\neg Q_{1}(x), \neg Q_{2}(x)\right\}\right\}$ and $P(f(f(f(x))))^{R}=\left\{\left\{Q_{1}(f(f(f(x))))\right\},\left\{Q_{1}(f(f(f(x))))\right\}\right\}$ respectively. Then $C_{2}{ }^{\prime}$ should be $Q_{1}(x) \wedge Q_{2}(x) \supset Q_{1}(f(x))$ with $\theta_{2}=\pi_{2}=\{f(f(x)) / x\}$, but $C_{1}{ }^{\prime}$ is either $Q_{1}(x) \wedge Q_{2}(x)$ $\supset Q_{1}(f(x))$ with $\theta_{1}=\pi_{1}=\{x / x\}$ or $Q_{1}(x) \wedge Q_{2}(x) \supset Q_{2}(f(x))$ with $\theta_{1}=\pi_{1}=\{x / x\}$. We set $C_{1}{ }^{\prime}=Q_{1}(x) \wedge Q_{2}(x) \supset Q_{1}(f(x))$ with $\theta_{1}=\pi_{1}=\{x / x\}$, and set $C_{1}{ }^{\prime \prime}=Q_{1}(x) \wedge Q_{2}(x) \supset$ $Q_{2}(f(x))$ with $\theta_{1}=\pi_{1}=\{x / x\}$. The set $C_{3}{ }^{R}=\left\{Q_{1}(x) \wedge Q_{2}(x) \supset Q_{1}(f(x)), Q_{1}(x) \wedge Q_{2}(x)\right.$ $\left.\supset Q_{2}(f(x))\right\}$. Both clauses should be used in the pseudo-subsumption by the reason in the proof. Therefore we set $C_{3}{ }^{\prime}=Q_{1}(x) \wedge Q_{2}(x) \supset Q_{1}(f(x))$ with $\pi_{3}=$ $\{f(x) / x\}$, and $C_{3}{ }^{\prime \prime}=Q_{1}(x) \wedge Q_{2}(x) \supset Q_{2}(f(x))$ with $\pi_{3}=\{f(x) / x\}$. By these clauses $C_{1}{ }^{\prime}, C_{1}{ }^{\prime \prime}, C_{2}{ }^{\prime}, C_{3}{ }^{\prime}, C_{3}{ }^{\prime \prime}$ and substitutions $\pi_{1}, \pi_{1}, \pi_{2}, \pi_{3}, \pi_{3}, C^{\prime}$ is pseudo subsumed by $C_{1}{ }^{\prime}$ and $C_{2}{ }^{\prime}$.

It is noted that in the above theorem the substitution does not change.

Definition 4.8. A set $S$ of clauses is regular if and only if $S$ has a simple satisfactory configuration $\left\langle S_{1}, \cdots, S_{2 N+1}\right\rangle$ such that its base set is $S$.

Proposition 4.9. Let $S_{m, n}$ be the set of all clauses which is pseudo-subsumed by some $n$ clauses in $S$ where the height of $\theta_{i}$ is less than or equal to $m$, then $S_{m, n}$ is a finite set. 
Proof. The number of clauses in $S$ is finite and the number of sets of clauses consisting of $n$ clauses of $S$ is finite. Also if height is limited, then instantiations of $C_{i}$ are essentially finite, and a pseudo-subsumed clause is constructed from those literals occurring in each instantiation of $C_{\imath}$. From these Proposition 4.9 is obvious.

It is noted that $S \subset S_{m, n}$ and $S_{m, n} \subset S_{m^{\prime}, n^{\prime}}$ if $m<m^{\prime}$ and $n<n^{\prime}$.

Proposition 4.10. If $S$ is a regular set of clauses, then for any $m$ and $n$, $S_{m, n}$ is regular.

Proof. By the assumption there exists a simple satisfactory configuration $\left\langle S_{1}, \cdots, S_{2 N+1}\right\rangle$ such that $S_{1}=S$ and $\left.S_{2 N+1}{ }^{R}\right\rangle S$. By Proposition 4.5, if $S_{2 \imath+1}$ is a quasi-contraction of $S_{22}$ and $S^{\prime} \subset\left(S_{2 i}\right)_{m, n}$, then there is a quasi-contraction $S^{\prime \prime}$ of $S^{\prime}$ which is a subset of $\left(S_{2 i+1}\right)_{m, n}$. By Proposition 4.6, if $S_{2(i+1)}$ is an expansion of $S_{2 i+1}$ and $S^{\prime} \subset\left(S_{2 i+1}\right)_{m, n}$, then $S^{\prime} \subset\left(S_{2 i}\right)_{m, n}$. By Theorem 4.7, if $S^{\prime} \subset\left(S_{2 N+1}\right)_{m, n}$, then $S^{\prime R} \subset\left(S_{2 N+1}{ }^{R}\right)_{m, n}$. This completes the proof.

For the function test 2 we have following propositions.

Proposition 4.11. If a set $S$ of clauses is regular by a flat transformation $R$ and $S^{\prime} \subset S$, and $L_{P}(R) \subset L_{P}\left(S^{\prime}\right)$, then the value of test $2\left(\left\langle S^{\prime}\right\rangle\right)$ is $S A T$.

Proof. By the assumption there exists a simple satisfactory configuration $\left\langle S_{1}, \cdots, S_{2 N+1}\right\rangle$ such that $S_{1}=S$ and $S_{2 N+1}{ }^{R} \gg S$. Because $\left\langle S^{\prime}, S^{\prime}, S^{\prime \uparrow}, \cdots, S^{\prime \uparrow N \uparrow}\right\rangle$ makes an elementary satisfactory configuration of $S^{\prime}$ by $R$ and $S$, the value of test $2\left(\left\langle S^{\prime}\right\rangle\right)$ is SAT.

Theorem 4.12. If a set $S$ of clauses is regulaa by a flat transformation $R, S^{\prime}$ is subsumed by $S$, and $\boldsymbol{L}_{\boldsymbol{P}}(R) \subset \boldsymbol{L}_{P}\left(S^{\prime}\right)$, then the value of test $2\left(\left\langle S^{\prime}\right\rangle\right)$ is $S A T$.

Proof. By the assumption it is obvious that there exists some $S_{m, n}$ such that for some quasi-contraction $S^{\prime \prime}$ of $S^{\prime}, S^{\prime \prime} \subset S_{m, n}$. Hence $S^{\prime \prime}$ has an elementary satisfactory configuration $\left\langle S^{\prime \prime}, S^{\prime \prime}, S^{\prime \prime \uparrow}, \cdots, S^{\prime \prime \uparrow N \uparrow}\right\rangle$ by $R$ and $S_{m, n}$ as the proof of Proposition 4.11. If we define $R^{\uparrow} \in\left[\boldsymbol{L}_{P}\left(S^{\prime \prime \uparrow N+1 \uparrow}\right) \rightarrow \boldsymbol{L}_{\boldsymbol{P}}\left(S^{\prime \prime \uparrow}\right)\right]$ by $P(\underline{x})^{\theta * R \uparrow}=$ $P(\underline{x})^{\theta R *}$, then $\left\langle S^{\prime \prime}, S^{\prime \prime}, S^{\prime \prime \uparrow}, \cdots, S^{\prime \prime \uparrow} \uparrow, S^{\prime \prime \uparrow N+1 \uparrow}\right\rangle$ is an elementary satisfactory configuration by $R^{\uparrow}$ and $S_{m, n}{ }^{\uparrow}$. Hence the value of test $2\left(\left\langle S^{\prime}\right\rangle\right)$ is SAT.

It is noted that in Theorem $4.2, S$ can be considered an open axiom of a theory, and therefore Theorem 4.2 means that if $S$ is regular, a set $S^{\prime}$ is provable from $S$ and all predicates of $S$ occur in $S^{\prime}$, then the value of test $2\left(S^{\prime}\right)$ is SAT. 


\section{$\S 5 . \quad$ An Equality Theory of Herbrand Universe}

Let $S$ be a set of clauses such that $L_{P}(S)=\{E\}, \mathbb{L}_{C}(S)=\{c\}, L_{F}(S)=\{f\}$ where $E$ and $f$ are binary. We define an interpretation $\boldsymbol{I}_{=}$to the Herbrand universe of $S$ setting that $E^{I}$ is the equality relation of Herbrand terms, $c^{I}$ is $c$ itself, $f^{I}$ is canonical that is $f^{I}(s, t)=f(s, t)$. We show in this section that if $S$ is true under the interpretation $\boldsymbol{I}_{=}$, that is, any clause in $S$ is true for arbitrary assignment of Herbrand terms for variables, then test $2(\langle S\rangle)$ takes the value SAT.

Proposition 5.1. A set $\mathbb{E}=\{E(x, x), E(x, y) \supset E(y, x), E(x, y) \wedge E(y, z) \supset$ $E(x, z), E(x, u) \wedge E(y, v) \supset E(f(x, y), f(u, v)), E(f(x, y), f(u, v)) \supset E(x, u) \wedge E(y, v)$, $\neg E(c, f(x, y))\}$ is regular.

Proof. The set $\mathbb{E}^{\uparrow}$ is $\left\{E_{c c}, E_{f f}\left(x_{1}, x_{2}, x_{1}, x_{2}\right), E_{c c} \supset E_{c c}, E_{c f}\left(y_{1}, y_{2}\right) \supset E_{f c}\left(y_{1}, y_{2}\right)\right.$, $E_{f c}\left(x_{1}, x_{2}\right) \supset E_{c f}\left(x_{1}, x_{2}\right), E_{f f}\left(x_{1}, x_{2}, y_{1}, x_{2}\right) \supset E_{f f}\left(y_{1}, y_{2}, x_{1}, x_{2}\right), \cdots, \neg E_{c f}\left(f\left(x_{1}, x_{2}\right)\right.$, $\left.\left.\left(y_{1}, y_{2}\right)\right)\right\}$. Let $R$ be a flat transformation from $\mathbb{L}_{P}\left(\mathbb{E}^{\uparrow}\right)$ to $\mathbb{L}_{P}(\mathbb{E})$ such that $E_{c c}{ }^{R}$ is true, $E_{c f}(x, y)^{R}$ is false, $E_{f c}(x, y)^{R}$ is false, and $E_{f f}(x, y, u, v)^{R}$ is $E(x, u) \wedge$ $E(y, v)$ respectively. By simple computation we can ascertain that $\mathbb{E}^{\uparrow} \gg \mathbb{E}$.

The set $\mathbb{E}$ of clauses may be considered as a basic theory of the equality in Herbrand universe, and is true under $\mathbb{k}_{=}$. We fix the flat transformation $R$ in this section as one in the proof of Proposition 5.1. Let $S$ be a set of clauses which has only one predicate $E$, and is true under $\mathbb{I}=$. If $S$ is subsumed by $\mathbb{E}$, then we have the result required in this section by Theorem 4.12 and Propsition 5.1, but this does not hold. Therefore we consider to extend $\mathbb{E}$ adding other true clauses so that any true set of clauses is subsumed. For example, let us consider $E \cup\{\neg E(x, f(x, y))\}$, then we have $\mathbb{E}^{\uparrow} \cup\left\{\neg E\left(x_{1}, f\left(x_{1}, x_{2}\right)\right) \vee\right.$ $\left.\neg E\left(x_{2}, c\right), \neg E\left(x_{1}, f\left(x_{1}, x_{2}\right)\right) \vee \neg E\left(x_{2}, f\left(y_{1}, y_{2}\right)\right)\right\}$. Hence the set $\mathbb{E} \cup\{\neg E(x, f(x, y))\}$ is also regular. Generalizing this we introduce the following definition and proposition.

Definition 5.2. An atom $E\left(x, f\left(t_{1}, t_{2}\right)\right)$ is said to be self contained if and only if $x$ has at least one occurrence in $t_{1}$ or $t_{2}$.

Let $\boldsymbol{E}_{n}$ be the set of clauses such that $\{\neg A \mid A$ is a self contained atom, and height $(A)<n\}$, and $\boldsymbol{E}_{\infty}$ is $\mathbb{E} \cup \bigcup_{i=1}{ }^{\infty} \mathbb{E}_{n}$. It is noted that $\neg A$ in $\mathbb{E}_{n}$ is true under $I_{=}$because for any substitution $\theta$, height $\left(x^{\theta}\right)<$ height $\left(f\left(t_{1}, t_{2}\right)^{\theta}\right)$ that means $x^{\theta} \neq f\left(t_{1}, t_{2}\right)^{\theta}$.

Proposition 5.3. The set $\mathbb{E} \cup \mathbb{E}_{n}$ of clauses is regular by $R$.

Proof. Let $\neg E\left(x, f\left(t_{1}, t_{2}\right)\right) \in \mathbb{E}_{n}$. If $\varepsilon$ is a decomposition such that $x^{\varepsilon}=c$, 
then $\left(\neg E\left(x, f\left(t_{1}, t_{2}\right)\right)\right)^{s}$ is $\left(\neg E\left(c, f\left(t_{1}{ }^{\varepsilon}, t_{2}{ }^{\varepsilon}\right)\right)\right)$ and $\left(\neg E\left(c, f\left(t_{1}{ }^{\varepsilon}, t_{2}{ }^{\varepsilon}\right)\right)\right)^{*}$ is $\neg E_{c f}\left(t_{1}{ }^{\varepsilon}, t_{2}{ }^{\varepsilon}\right)$. Hence $\left(\neg E_{c f}\left(t_{1}{ }^{s}, t_{2} \varepsilon\right)\right)^{R}$ is true. Otherwise if $\varepsilon$ is a decomposition such that $x^{\varepsilon}=f\left(x_{1}, x_{2}\right)$, then $\left(\neg E\left(x, f\left(t_{1}, t_{2}\right)\right)\right)^{\varepsilon}$ is $\neg E\left(f\left(x_{1}, x_{2}\right), f\left(t_{1}{ }^{\varepsilon}, t_{2}{ }^{\varepsilon}\right)\right)$. Hence $\left(\neg E\left(f\left(x_{1}, x_{2}\right)\right.\right.$, $\left.\left.f\left(t_{1}{ }^{\varepsilon}, t_{2}{ }^{\varepsilon}\right)\right)\right)^{* R}$ is $\neg E\left(x_{1}, t_{1}^{\varepsilon}\right) \vee \neg E\left(x_{2}, t_{2}{ }^{\varepsilon}\right)$. By the assumption $E\left(x_{1}, t_{1}{ }^{\varepsilon}\right)$ or $E\left(x_{2}, t_{2}{ }^{\varepsilon}\right)$ is self contained and have the height less than or equal to $n$, and therefore it is contained in $\boldsymbol{E}_{n}$.

Propositions 5.4, 5.5 and 5.6 are preparations for Theorem 5.9 which shows that any clause true under $\boldsymbol{I}_{=}$is subsumed by $\boldsymbol{E}_{\infty}$.

Proposition 5.4. Let $\theta$ be an identity substitution except $x^{\theta}=t$, then $E(x, t)$ $\wedge E\left(s_{1}{ }^{\theta}, s_{2}{ }^{\theta}\right) \supset E\left(s_{1}, s_{2}\right)$ is subsumed by $\boldsymbol{E}$.

Proof. First we show $E(x, t) \supset E\left(s^{\theta}, s\right)$ by the induction on the height of the term $s$. For height 1 , if $s \equiv c$ then $E(x, t) \supset E(c, c)$ is obvious, if $s \equiv y$ and $x \neq y$, then $E(x, t) \supset E(y, y)$ is obvious, if $s \equiv x$ then $E(x, t) \supset E(t, x)$ is the symmetry axiom. For $s=f\left(t_{1}, t_{2}\right)$, we can assume $E(x, t) \supset E\left(t_{1}{ }^{\theta}, t_{1}\right)$ and $E(x, t) \supset$ $E\left(t_{2}{ }^{\theta}, t_{2}\right)$ as the induction hypothesis, and $E\left(t_{1}{ }^{\theta}, t_{1}\right) \wedge E\left(t_{2}{ }^{\theta}, t_{2}\right) \supset E\left(f\left(t_{1}{ }^{\theta}, t_{2}{ }^{\theta}\right), f\left(t_{1}, t_{2}\right)\right)$ is an instantiation of $E(x, u) \wedge E(y, v) \supset E(f(x, y), f(u, v))$, and therefore we have easily the required formula. Next, because we have shown that $E(x, t) \supset E\left(s_{1}{ }^{\theta}, s_{1}\right)$ and $E(x, t) \supset E\left(s_{2}{ }^{\theta}, s_{2}\right)$, we have easily $E(x, t) \wedge E\left(s_{1}{ }^{\theta}, s_{2}{ }^{\theta}\right) \supset E\left(s_{1}, s_{2}\right)$ using the transitive and the symmetry axioms in $\boldsymbol{E}$.

Proposition 5.5. Let $\theta$ be an identity substitution except $x^{\theta}=t$, then $E(x, t)$ $\wedge \neg E\left(s_{1}{ }^{\theta}, s_{2}{ }^{\theta}\right) \supset \neg E\left(s_{1}, s_{2}\right)$ is subsumed by $\boldsymbol{E}$.

Proof. The given formula is actually $\neg E(x, t) \vee E\left(s_{1}{ }^{\theta}, s_{2}{ }^{\theta}\right) \vee \neg E\left(s_{1}, s_{2}\right)$ in the clausal form. The given clause is rewritten as $E(x, t) \wedge E\left(s_{1}, s_{2}\right) \supset E\left(s_{1}{ }^{\theta}, s_{2}{ }^{\theta}\right)$. In the proof of Proposition 5.4, we have shown $E(x, t) \supset E\left(s^{\theta}, s\right)$, so that $E(x, t)$ $\supset E\left(s, s^{\theta}\right)$. The given clause is easily subsumed by $\boldsymbol{E}$.

Proposition 5.6. Let $\theta$ be an identity substitution except $x^{\theta}=t$, and $C=$ $L_{1} \vee \cdots \vee L_{n}$, then $E(x, t) \wedge L_{\imath}{ }^{\theta} \supset C$ is subsumed by $\boldsymbol{E}$ for any $i$.

Proof. By Propositions 5.3 and 5.4, we have $E(x, t) \wedge L_{\imath}{ }^{\theta} \supset L_{i}$, and therefore Proposition 5.6 is obvious.

Roughly speaking by Proposition 5.6 we have that $E(x, t) \wedge C^{\theta} \supset C$ is subsumed by $\boldsymbol{E}$.

Definition 5.7. An atom $A$ is said to be normal if and only if either (1) $A$ is of the form $E(x, t)$ such that $x$ does not occur in $t$, or (2) $A$ is of the form $E(s, t)$ such that $t$ does not contain any variable, and height $(s)<$ height $(t)$ if neither $s$ nor $t$ contains variables. A clause $C$ is said to be normal if and only 
if $C$ does not contain the negation symbol and any atom is normal.

Lemma 5.8. Any normal clause $C$ is false under the interpretation $\boldsymbol{I}_{=}$.

Proof. We use the induction on the number of variables occurring in $C$. If $C$ does not contain any variable then any atom is of the form $E(s, t)$ such that $s, t$ are Herbrand terms. Hence by the definition of the normal clause, $C$ is obviously false under $\mathbb{I}=$. Let $C[z]$ be a clause which contains a variable $z$ and let $u$ be a Herbrand term such that height $(u)>$ height $(C)$. We show that $C[u / z]$ is converted to a normal clause $C^{\prime}$ not changing the truth value and the number of variables. If $A \in C$ is of the form $E(x, t)$ such that $x$ is distinct from $z$, then $E(x, t)[u / z]$ obviously satisfies the condition of normal atoms. If $E(z, t) \in C$, then $E(z, t)[u / z]$ is $E(u, t)$ and this is converted to $E(t, u)$. This conversion does not change the truth value and the number of variables, and height $(t)<$ height $(u)$ by the choice of $u$. If $E(s, t)$ is an atom of $C$ which satisfies the last condition of normal atom and contains the variable $z$, then $E(s, t)[u / z]$ is $E(s[u / z], t)$. If $s[u / z]$ contains another variable then $E(s[u / z], t)$ is normal, else $E(t, s[u / z])$ is normal.

Theorem 5.9. If a clause $C$ which consists of only one predicate symbol $E$ is true under $\mathbb{I}_{=}$, then $C$ is subsumed by $\mathbb{E}_{\infty}$.

Proof. We show by the induction on the number of variables occurring in $C$ and the sum of heights of terms in $C$. If $C=E\left(f\left(s_{1}, t_{1}\right), f\left(s_{2}, t_{2}\right)\right) \vee D$ is true under $\mathbb{I}_{=}$, then $C^{\prime}=E\left(s_{1}, s_{2}\right) \vee D$ and $C^{\prime \prime}=E\left(t_{1}, t_{2}\right) \vee D$ are both true under $\mathbb{I}_{=}$, and the sum of heights of terms reduces. If $C^{\prime}$ and $C^{\prime \prime}$ are subsumed by $\boldsymbol{E}_{\infty}$ then $C$ is obviously subsumed by $\boldsymbol{E}_{\infty}$. If $C=\neg E\left(f\left(s_{1}, t_{1}\right), f\left(s_{2}, t_{2}\right)\right) \vee D$ is true under $\boldsymbol{I}_{=}$, then $C^{\prime \prime}=\neg E\left(s_{1}, s_{2}\right) \bigvee \neg E\left(t_{1}, t_{2}\right) \bigvee D$ is true under $\boldsymbol{I}_{=}$, and the sum of heights of terms reduces. If $C^{\prime}$ is subsumed by $\boldsymbol{E}_{\infty}$ then $C$ is obviously subsumed by $\boldsymbol{E}_{\infty} . \quad$ By the above two facts we can assume that if an atom $E(s, t)$ is contained in $C$, then height $(s)$ or height $(t)$ is 1 . Furthermore by the similar argument we can assume that height $(s)$ is 1 .

We consider an atom of the form $E\left(c, f\left(t_{1}, t_{2}\right)\right)$. If $C=E\left(c, f\left(t_{1}, t_{2}\right)\right) \vee D$ is true under $\boldsymbol{I}_{=}$, then $D$ is true under $\mathbb{I}_{=}$, and the sum of heights of terms reduces. If $D$ is subsumed by $\boldsymbol{E}_{\infty}$ then $C$ is obviously subsumed by $\boldsymbol{E}_{\infty}$. A clause of the form $\neg E\left(c, f\left(t_{1}, t_{2}\right)\right) \vee D$ is true under $\boldsymbol{I}_{=}$, and $\neg E\left(c, f\left(t_{1}, t_{2}\right)\right)$ is subsumed by $\boldsymbol{E}_{\infty}$ so that $\neg E\left(c, f\left(t_{1}, t_{2}\right)\right) \vee D$ is subsumed by $\boldsymbol{E}_{\infty}$.

We consider an atom of the form $E\left(x, f\left(t_{1}, t_{2}\right)\right)$ such that $x$ occurs in $t_{1}$ or $t_{2}$. This is a self contained atom, so can be treated by a similar argument as above. For atoms of the form $E(x, x)$ or $E(c, c)$, we can treat similarly.

Hence we can assume that any atom in $C$ is of the form $E(x, t)$ such that $t$ does not contain $x$. If $C$ contains a literal $\neg E(x, t)$ such that $t$ does not contain $x$, then $C$ can be rewritten as $E(x, t) \supset D[x]$. Therefore $D[t / x]$ is true 
under $\boldsymbol{J}_{=}$, and subsumed by $\boldsymbol{E}_{\infty}$ by the induction hypothesis. By Proposition 5.6 and the fact that $D[t / x]$ subsumed by $\boldsymbol{E}_{\infty}, E(x, t) \supset D[x]$ is subsumed by $\boldsymbol{E}_{\infty}$. If $C$ does not contain a negation symbol, then $C$ is a normal clause, and false under $\boldsymbol{I}_{\boldsymbol{}}$ by Lemma 5.8 .

Theorem 5.10. If a set $S$ of clauses such that $L_{P}(S)=\{E\}, L_{C}(S)=\{c\}$ and $\boldsymbol{L}_{\boldsymbol{F}}(S)=\{f\}$ where $E$ and $f$ are binary is true under the interpretation $\boldsymbol{I}_{=}$, then test $2(\langle S\rangle)$ takes the value $S A T$.

Proof. By Theorem 5.9, $S$ is subsumed by $\boldsymbol{E}_{\infty}$. Therefore $S$ is subsumed by some $\boldsymbol{E} \cup \boldsymbol{E}_{n}$ by the compactness. The set $\boldsymbol{E} \cup \boldsymbol{E}_{n}$ of clauses is regular. Hence by Theorem 4.12 , test $2(\langle S\rangle)$ takes the value SAT.

For example we consider a set $S$ of clauses $\{E(x, x), \neg E(x, f(y, c)) \vee$ $\neg E(y, f(x, c))\}$ which is true under $\boldsymbol{I}_{=}$. We set $S_{1}=S$.

The set $S_{2}=S_{1}{ }^{\uparrow}$ is $\left\{E_{c c}, E_{f f}\left(x_{1}, x_{2}, x_{1}, x_{2}\right), \neg E_{c f}(c, c) \vee \neg E_{c f}(c, c), \neg E_{c f}\left(f\left(y_{1}, y_{2}\right), c\right)\right.$ $\vee \neg E_{f f}\left(y_{1}, y_{2}, c, c\right), \neg E_{f f}\left(x_{1}, x_{2}, c, c\right) \vee \neg E_{c f}\left(f\left(x_{1}, x_{2}\right), c\right), \neg E_{f f}\left(x_{1}, x_{2}, f\left(y_{1}, y_{2}\right), c\right) \vee$ $\left.\neg E_{f f}\left(y_{1}, y_{2}, f\left(x_{1}, x_{2}\right), c\right)\right\}$. Then $S_{2}{ }^{R}$ becomes $S_{3}=\left\{E(x, x), \neg E\left(x_{1}, f\left(y_{1}, y_{2}\right)\right) \bigvee\right.$ $\left.\neg E\left(x_{2}, c\right) \vee \neg E\left(y_{1}, f\left(x_{1}, x_{2}\right)\right) \vee \neg E\left(y_{2}, c\right)\right\}$. We take a quasi-contraction $S_{4}$ of $S_{1} \cup S_{3}$ such that $S_{4}=S_{1} \cup\{\neg E(x, f(y, v)) \vee \neg E(y, f(x, u))\}$. The set $S_{5}=S_{4}{ }^{\uparrow}$ is $S_{2} \cup$ $\left\{\neg E_{c f}(c, c) \vee \neg E_{c f}(c, c), \neg E_{c f}\left(c, f\left(v_{1}, v_{2}\right)\right) \vee \neg E_{c f}(c, c), \cdots, \neg E_{f f}\left(x_{1}, x_{2}, f\left(y_{1}, y_{2}\right), f\left(v_{1}, v_{2}\right)\right)\right.$ $\vee \neg E_{f f}\left(y_{1}, y_{2}, f\left(x_{1}, x_{2}\right), c\right), \neg E_{f f}\left(x_{1}, x_{2}, f\left(y_{1}, y_{2}\right), c\right) \vee \neg E_{f f}\left(y_{1}, y_{2}, f\left(x_{1}, x_{2}\right), f\left(u_{1}, u_{2}\right)\right)$, $\left.\neg E_{f f}\left(x_{1}, x_{2}, f\left(y_{1}, y_{2}\right), f\left(v_{1}, v_{2}\right)\right) \wedge \neg E_{f f}\left(y_{1}, y_{2}, f\left(x_{1}, x_{2}\right), f\left(u_{1}, u_{2}\right)\right)\right\}$. Then $S_{5}{ }^{R}$ becomes $S_{6}=S_{3} \cup\left\{\neg E\left(x_{1}, f\left(y_{1}, y_{2}\right)\right) \vee \neg E\left(x_{2}, f\left(v_{1}, v_{2}\right)\right) \vee \neg E\left(y_{1}, f\left(x_{1}, x_{2}\right)\right) \vee \neg E\left(y_{2}, c\right)\right.$, $E\left(x_{1}, f\left(y_{1}, y_{2}\right)\right) \vee \neg E\left(x_{2}, c\right) \vee \neg E\left(y_{1}, f\left(x_{1}, x_{2}\right)\right) \vee \neg E\left(y_{2}, f\left(u_{1}, u_{2}\right)\right), E\left(x_{1}, f\left(y_{1}, y_{2}\right)\right) \vee$ $\left.\neg E\left(x_{2}, f\left(v_{1}, v_{2}\right)\right) \vee \neg E\left(y_{1}, f\left(x_{1}, x_{2}\right)\right) \vee \neg E\left(y_{2}, f\left(u_{1}, u_{2}\right)\right)\right\}$. It is obvious that $S_{4} \ll S_{6}$. Therefore $\left\langle S_{1}, S_{1}, S_{2}\right\rangle$ is an elementary satisfactory configuration by $R$ and a saturated set $S_{4}$.

The conditions $\boldsymbol{L}_{\boldsymbol{C}}(S)=\{c\}$ and $\boldsymbol{L}_{\boldsymbol{F}}(S)=\{f\}$ are only for the sake of brevity, and the case that $S$ has other constants and functions can be treated by the similar arguments to those in this section.

\section{$\S 6$. The Sets of Clauses Satisfiable in Finite Domain}

In this section we show that if a set $S$ of clauses is satisfiable under an interpretation $\boldsymbol{I}$ with finite domain $\boldsymbol{D}$, then test $2(\langle S\rangle)$ takes the value SAT. We consider a property of the occurrence of a variable. Let $x^{0}$ be an occurrence of a variable $x$ in a literal, then the level of $x^{0}$ is defined as usual and is denoted by level $\left(x^{0}\right)$.

Definition 6.1. A variable $x$ occurs at level 1 in $x$ itself, and if $x$ occurs at level $n$ in term $t$, then the occurrence has level $n+1$ in term $f\left(\underline{s}_{1}, t, \underline{s}_{2}\right)$ and has level $n$ in literal $P\left(\underline{s}_{1}, t, \underline{s}_{2}\right)$ or $\neg P\left(\underline{s}_{1}, t, \underline{s}_{2}\right)$. The level of $x^{0}$ in a clause or 
set of clauses is defined by that for the literal in which $x^{0}$ occurs.

With respect to a configuration the ancestor and descendant relation between variables is defined as follows.

Definition 6.2. For a variable $x$ and a decomposition operation $\{f(\underline{y}) / x, \cdots\}$, each variable in $\underline{y}$ is said to be a child of $x$. For either of a predicate decomposition, a quasi-contraction or a flat transformation, the child of $x$ is $x$ itself if it remains in the result of the operation. The descendants of a variable is defined as usual.

Proposition 6.3. If a variable $x$ occurs at level $n$, any descendant variable of $x$ also occurs at the same level $n$ after either of a expansion, a quasi-contraction or a flat transformation.

Proof. For the expansion, the operation is divided in two parts. One is the decomposition and this operation increases the level by one, the other is the predicate decomposition and this decreases that by one. The quasi-contraction and the flat transformation do not change the level of occurrence except that these may erase the occurrence.

For example let $C$ be $P(x, f(y))$, and $C^{\prime}$ be $P_{c f}\left(f\left(y_{1}\right)\right) \in C^{\uparrow}$ where $C^{\prime}$ is the result of a decomposition $\left\{c / x, f\left(y_{1}\right) / y\right\}$, the child of $x$ does not occur in $C^{\prime}$ and the child $y_{1}$ of $y$ has the same level 2 as that of $y$.

Definition 6.4. Let $x^{0}$ and $y^{0}$ be two occurrences of variables in a clause $C$, and $X=\left\{\right.$ height $(s)-1 \mid s$ contains both $x^{0}$ and $y^{0}$ where $s$ is a term in $\left.C\right\}$ then the distance between $x^{0}$ and $y^{0}$ which is denoted by $d_{C}\left(x^{0}, y^{0}\right)$ is defined by $d_{C}\left(x^{0}, y^{0}\right)=$ minimum $X$ if $X$ is not empty and otherwise infinite.

Proposition 6.5. Let $x^{0}$ and $y^{0}$ be occurrences of variables in a clause $C$ such that level $\left(x^{0}\right)>1$, then the distance between two children of $x^{0}$ in $C^{\uparrow}$ is 1 , and for a child $x^{\prime 0}$ of $x^{0}$ and a child $y^{\prime 0}$ of $y^{0}, d_{C \uparrow}\left(x^{\prime 0}, y^{\prime 0}\right)$ is $d_{C}\left(x^{0}, y^{0}\right)+1$ if $d_{C \uparrow}\left(x^{\prime 0}, y^{\prime 0}\right)$ is finite.

Proof. Let $\theta=\{f(\underline{x}) / x, \cdots\}$ be a decomposition operation for $x$, and if level $\left(x^{0}\right)>1$, then $x^{0 \theta}$ remains unchanged through predicate decomposition operations. Let $s^{\theta}$ be the minimum height term which contains both $x^{\prime 0}$ and $y^{\prime 0}$, then $s$ is the term of the minimum height that contains both occurrences $x^{0}$ and $y^{0}$.

For example let $C$ be $P(c, f(f(x, y), c))$, and $C^{\prime}$ be $P_{c f}\left(f\left(f\left(x_{1}, x_{2}\right), f\left(y_{1}, y_{2}\right)\right), c\right)$ $\in C^{\uparrow}$ where $C^{\prime}$ is the result of a decomposition $\left\{f\left(x_{1}, x_{2}\right) / x, f\left(y_{1}, y_{2}\right) / y\right\}$, then $d_{C^{\prime}}\left(x_{1}, x_{2}\right)$ is 1 by $f\left(x_{1}, x_{2}\right)$, and $d_{C^{\prime}}\left(x_{1}, y_{1}\right)$ is 2 by $f\left(f\left(x_{1}, x_{2}\right), f\left(y_{1}, y_{2}\right)\right)$ where $d_{C}(x, y)$ is 1 by $f(x, y)$. 
Proposition 6.6. Let $m=$ height $(S)$, then after at least $m$ expansions, any term becomes a substitution instance of some variable.

Proof. Any term $t$ of height $(t) \leqq i$ in $S^{\uparrow i \uparrow}$ which contains at least one variable, is a substitution instance of some variable which is the height $(t)$-th ancestor of that variable because of Proposition 6.5. The originally constant term goes to a predicate symbol and disappears after $m$ expansions as a term.

Proposition 6.7. Let $m$ be height $(S)$. In each term of $S^{\uparrow}{ }^{n \uparrow}(n \geqq m)$, the level of the occurrences of variables are the same. It two terms of $S^{\uparrow n \uparrow}$ have occurrences of the same variable, then one term has the occurrence of the other as its subterm.

Proof. In the substitution instance for a variable of composition of decomposition substitutions, any variable occurs at the same level. And if two terms have occurrences of the same variable, then the two variables corresponding these terms are in the relation of ancestor and descendant. This means that one term includes the other.

For example let $S$ be $\{P(x, f(f(x, y), f(y, c)))\}$, and $C^{\prime}$ be $P_{f f, f c f f, f c f f}\left(x_{111}, x_{112}\right.$, $\left.f\left(f\left(x_{111}, x_{112}\right), c\right), c, f\left(f\left(y_{111}, y_{112}\right), c\right), c\right) \in S^{\uparrow \uparrow \uparrow}$ where $C^{\prime}$ is the result of a repetition $\left\{f\left(x_{1}, x_{2}\right) / x, f\left(y_{1}, y_{2}\right) / y\right\},\left\{f\left(x_{11}, x_{12}\right) / x_{1}, c / x_{2}, f\left(y_{11}, y_{12}\right) / y_{1}, c / y_{2}\right\}$ and $\left\{f\left(x_{111}, x_{112}\right)\right.$ $\left./ x_{11}, c / x_{12}, f\left(y_{111}, y_{112}\right) / y_{11}, c / y_{12}\right\}$ of decompositions, and predicate decompositions. For Proposition 6.6, it is enough to consider two terms $f\left(f\left(x_{111}, x_{112}\right), c\right)$, $f\left(f\left(y_{111}, y_{112}\right), c\right)$ which are substitution instances of $x_{1}$ and $y_{1}$ respectively. For Proposition 6.7 , the variable $x_{111}$ has two occurrences with different level in $C^{\prime}$, but they are not in the same term.

It is noted that if there is an interpretation $\boldsymbol{I}$ of a set $S$ of clauses, then $\boldsymbol{I}$ can be canonically extended to any $S^{\uparrow i \uparrow}$, that is, if $P_{f 1, \ldots, f n}\left(\underline{x}_{1}, \cdots, \underline{x}_{n}\right)$ is the decomposed predicate for $P\left(f_{1}\left(\underline{x}_{1}\right), \cdots, f_{n}\left(\underline{x}_{n}\right)\right)$, then we set $P_{f 1, \ldots, f n}{ }^{I}\left(\underline{d}_{1}, \cdots, \underline{d}_{n}\right)$ $=P^{I}\left(f_{1}{ }^{I}\left(\underline{d}_{1}\right), \cdots, f_{n}{ }^{I}\left(\underline{d}_{n}\right)\right)$.

Definition 6.8. For $R \in\left(\boldsymbol{L}_{\boldsymbol{P}}\left(S^{\uparrow{ }^{\natural}}\right) \rightarrow \boldsymbol{L}_{\boldsymbol{P}}(S)\right)$, and an interpretation $\boldsymbol{I}$ of $S, R$ is said to be compatible with $\boldsymbol{I}$ if and only if for any atom $A \in S^{\uparrow k \uparrow}$ it holds that $A^{I}=\left(A^{R}\right)^{I}$.

Definition 6.9. A transformation $R \in\left(\boldsymbol{L}_{\boldsymbol{P}}\left(S^{\uparrow k \uparrow}\right) \rightarrow \boldsymbol{L}_{\boldsymbol{P}}(S)\right)$, is said to be $p$ seudo flat if and only if any variable occurs at top level in $P(\underline{x})^{R}$ for any $P \in \boldsymbol{L}_{\boldsymbol{P}}\left(S^{\uparrow k \uparrow}\right)$.

Definition 6.10. Let $\left\langle S_{1}, \cdots, S_{2 N+1}\right\rangle$ be a configuration. This is said to be a pseudo elementary satisfactory configuration if it satisfies the following conditions :

(1) For some $K<N$, there exists a pseudo flat transformation $R \in\left(\boldsymbol{L}_{\boldsymbol{P}}\left(S_{2 N+1}\right)\right.$ $\left.\rightarrow \boldsymbol{L}_{\boldsymbol{P}}\left(S_{2 K+1}\right)\right)$, 
(2) There exists a set $S_{2 K+1}{ }^{\prime}$ of clauses such that it includes $S_{2 K+1}$ and i. satisfies $S_{2 K+1}{ }^{\prime \uparrow . N-K \uparrow R} \gg S_{2 K+1}{ }^{\prime}$.

Proposition 6.11. If $S$ has a pseudo elementary satisfactory configuration, then $S$ has an elenentary satisfactory configuration.

Proof. Let $\left\langle S_{1}, \cdots, S_{2 N+1}\right\rangle$ be a pseudo elementary satisfactory sequence and $R \in\left(S^{\uparrow k \uparrow} \rightarrow S\right)$ be a pseudo flat transformation such that $S_{2 K+1}{ }^{1 \uparrow N-K \uparrow R} \gg S_{2 K+1}{ }^{\prime}$. We put $S_{2 N+2}=S_{2 N+3}=S_{2 N+1}$, and define $R^{\uparrow} \in\left(\boldsymbol{L}_{\boldsymbol{P}}\left(S_{2 N+3}\right) \rightarrow \boldsymbol{L}_{\boldsymbol{P}}\left(S_{2 K+3}\right)\right)$ by $P(\underline{x})^{\theta * R \uparrow}$ $=P(\underline{x})^{\theta R *}$. Then obviously $R^{\uparrow}$ is a pseudo flat transformation and the height of constants decreases by one. Further, $\left.S_{2 K+1}{ }^{\prime} \uparrow \uparrow N-K \uparrow R\right\rangle S_{2 K+1}{ }^{\prime \uparrow}$ and $S_{2 K+3}$ is obviously included in $S_{2 K+1}{ }^{\prime}$. Hence Proposition 6.11 is obvious.

Definition 6.12. A pseudo flat transformation $R$ is said to be pseudo monadic if any atom in $P(\underline{x})^{R}$ has at most one occurrence of variable.

Definition 6.13. Two terms in a clause are said to have the property $A$ if they satisfy the condition that if each has an occurrence of the same variable then one has an occurrence of the other as its subterm. For the latter case we say that one includes the other. A clause is said to have the property $A$ if every pair of terms has the property $A$.

Proposition 6.14. If $S$ has the property $A$, then both $S^{\uparrow}$ and $S^{R}$ have the property $A$ where $R$ is a pseudo flat transformation.

Proof. For $S^{\uparrow}$, if $s^{\prime} \in s^{\theta *}$ and $t^{\prime} \in t^{\theta *}$ have the occurrences of the same variable then by the definition of a decomposition $s$ and $t$ have the occurrences of the same variable, and therefore we can assume that $s$ includes $t$. If $s$ has an occurrence of $t$ as its proper subterm, then obviously $s^{\prime}$ has an occurrence of $t^{\prime}$ as its proper subterm, and if $s=t$, then obviously $s^{\prime}=t^{\prime}$. For the transformation $R$, the terms in $S^{R}$ also appear in $S$ except constant terms.

Proposition 6.15. For a set $S$ of clauses, if there is an interpretation $I$ of $S$ which satisfies $S$, and there is a pseudo flat transformation $R \in\left(\mathbb{L}_{P}\left(S^{\uparrow k \uparrow}\right) \rightarrow\right.$ $\left.\boldsymbol{L}_{\boldsymbol{P}}(S)\right)$ which is conpatible with $\boldsymbol{I}$ and pseudo monadic, then test $2(\langle S\rangle)$ takes the value $S A T$.

Proof. It is enough to show that $S$ has a pseudo elementary satisfactory configuration. A clause $C$ is said to be elementary true if it is true under the interpretation $\boldsymbol{I}$, it has the property $A$, and each literal in $C$ has at most one argument with variable occurrences. Let $S_{m}$ be the set of all elementary true clauses $C$ of $L(S)$ such that height $(C) \leqq m$ and no proper subset of $C$ is true. It is obvious that $S_{m}$ is finite, because any variable in $C$ should also occur in a term by the property $A$. Let $m>\max$ (height $(R)$, height $(S)$ ), then obviously 
$\boldsymbol{S}_{m}{ }^{\uparrow \uparrow \uparrow R} \gg \boldsymbol{S}_{m}$ by Proposition 6.14. Let $A_{0}$ and $B_{0}$ be $S, A_{n+1}$ be $A_{n}{ }^{\uparrow \uparrow R} \cup S$ and $B_{n+1}$ be $B_{n}^{\uparrow k \uparrow R}$, then $A_{m}=B_{0} \cup \cdots \cup B_{m}$ is subsumed by $A_{m} \cup \boldsymbol{S}_{m}$ because if $i<m$ then $B_{i}$ is included in $A_{m}$, and $B_{m}$ is subsumed by $\boldsymbol{S}_{m}$ by Proposition 6.7 and by the fact that $R$ is pseudo monadic. Hence obviously $\left(A_{m} \cup \boldsymbol{S}_{m}\right)^{\uparrow k \uparrow R} \gg\left(A_{m} \cup \boldsymbol{S}_{m}\right)$ and $S \subset\left(A_{m} \cup \boldsymbol{S}_{m}\right)$. For any $i,\left(A_{m} \cup \boldsymbol{S}_{m}\right)^{\uparrow i \uparrow R}$ does not contain a propositional contradictory instance because this set is true under the interpretation $\boldsymbol{I}$. This means that $S$ has a pseudo elementary satisfactory configuration.

Let $S$ be a set of clauses which is satisfiable by some interpretation $\boldsymbol{I}$ with domain $\boldsymbol{D}$ of a finite number $N$ elements. Any term in Herbrand universe is interpreted by $\boldsymbol{I}$ as an element of $\boldsymbol{D}$. If this correspondence is not onto then we can restrict it to the image of $\boldsymbol{I} . \boldsymbol{I}^{-1}(d)$ denotes a Herbrand term with the smallest height among the Herbrand terms interpreted by $\boldsymbol{I}$ as $d \in \boldsymbol{D}$.

Definition 6.16. Let $\left\langle d, d^{\prime}\right\rangle$ be a pair of elements of $\boldsymbol{D}$, and let $\delta$ designate either $\neg$ or empty, then $\kappa_{\left\langle d, d^{\prime}\right\rangle}(x)$ denotes $\delta P\left(\underline{t}_{1}, x, \underline{t}_{2}\right)$ where $P \in \boldsymbol{L}_{P}(S), \underline{t}_{i}$ is a sequence of inverse images of a sequence $\underline{d}_{i}$ of elements of $D, \delta P^{I}\left(\underline{d}_{1}, d, \underline{d}_{2}\right)$ is true, $\delta P^{I}\left(\underline{d}_{1}, d^{\prime}, \underline{d}_{2}\right)$ is false, and if there is no such $P^{I}\left(\underline{d}_{1}, x, \underline{d}_{2}\right)$ then $\kappa_{\left\langle d, d^{\prime}\right\rangle}(x)$ denotes true.

Definition 6.17. For $d \in D, \lambda_{d}(x)$ denotes $\bigwedge_{d^{\prime} \in D^{\prime}} \kappa_{\left\langle d, d^{\prime}\right\rangle}(x)$.

Proposition 6.18. Any predicate $P \in L_{P}(S)$ is represented by $P\left(x_{1}, \cdots, x_{n}\right) \equiv$ $\bigvee_{\left\langle d_{1}, \ldots, d_{n}\right\rangle \in P \boldsymbol{I}} \wedge_{d_{i}} \lambda_{d_{i}}\left(x_{2}\right)$, that is, this formula is true under the interpretation $\boldsymbol{I}$.

Proof. For $\left\langle d_{1}, \cdots, d_{n}\right\rangle \in P^{I}$ if $\left\langle e_{1}, \cdots, e_{n}\right\rangle$ is a sequence such that $\lambda_{d_{i}}{ }^{I}\left(e_{i}\right)$ holds for any $i$, then obviously $P^{I}\left(e_{1}, d_{2}, \cdots, d_{n}\right)$ is true and so on. Hence $P^{I}\left(e_{1}, \cdots, e_{n}\right)$ is true.

The predicate $\lambda_{d}{ }^{I}$ is a subset of $\boldsymbol{D}$ which contains $d$, so that $\left\langle\lambda_{d_{1}}{ }^{I}, \cdots, \lambda_{d_{N}}{ }^{I}\right\rangle$ is an element of finite set $\boldsymbol{D}^{N}$.

Theorem 6.19. If a set $S$ of clauses is satisfiable under an interpretation $\boldsymbol{I}$ with finite domain $\boldsymbol{D}$, then test $2(\langle S\rangle)$ takes the value $S A T$.

Proof. There is a sequence $\left\langle\lambda_{d_{1}}{ }^{I}, \cdots \lambda_{d_{N}}{ }^{I}\right\rangle$ for each $S^{\uparrow i \uparrow}$, and consequently for some $i<j$ these coincide. From $S^{\uparrow \jmath \uparrow}$ to $S^{\uparrow i \uparrow}$ there is a canonical pseudo flat transformation $R$ such that $P^{\uparrow i \uparrow}\left(x_{1}, \cdots, x_{n}\right)^{R} \equiv \bigvee_{\left\langle d_{1}, \ldots, d_{n}\right\rangle \in P \uparrow i \uparrow I} \wedge_{d_{i}} \lambda_{d_{i}}\left(x_{i}\right)$ where $P \in L_{P}\left(S^{\uparrow j \uparrow}\right)$ and $\lambda_{d}$ is that of $S^{\uparrow i \uparrow}$. This transformation is pseudo monadic and compatible with $I$ from the construction. Therefore by Proposition 6.15 we have our theorem.

For example we consider a formula $[\forall x(P(x) \supset \neg P(f(x))) \wedge \forall x(P(x) \supset$ $P(f(f(f(x)))))] \supset \forall x(\neg P(x))$. This formula is false in a domain"w which have at 
least three elements. We fix an interpretation $\boldsymbol{I}$ with ia domain $\boldsymbol{D}=\left\{d_{0}, d_{1}, d_{2}\right\}$, and set $f^{I}\left(d_{2}\right)=d$, where $j=i+1 \bmod 3$, and $P^{I}(x)$ is true if and only if $x=d_{0}$. The given formula is false under this interpretation.

The clausal form becomes $S_{1}=\{P(x) \supset \neg P(f(x)), P(x) \supset P(f(f(f(x)))), P(c)\}$. The interpretation $\mathbb{l}$ can be extended to $S_{1}$ setting $c^{I}=d_{0}$. The set $S_{1}$ is obviously true under this interpretation 1 . The set $S_{1}$ has only one monadic predicate $P$, and it is obvious that $\lambda_{d_{0}}(x)=P(x), \lambda_{d_{1}}(x)=\neg P(x)$, and $\lambda_{d_{2}}(x)=$ $\neg P(x)$.

The expansion of $S_{1}$ is $S_{2}=\left\{P_{c} \supset \neg P_{f}(c), P_{f}(x) \supset \neg P_{f}(f(x)), P_{c} \supset P_{f}(f(f(c)))\right.$, $\left.P_{f}(x) \supset P_{f}(f(f(f(x)))), P_{c}\right\}$. We take a quasi-contraction $S_{3}$ of $S_{2}$ such that $S_{3}=$ $\left\{P_{c}, \neg P_{f}(c), P_{f}(f(f(c))), P_{f}(x) \supset \neg P_{f}(f(x)), P_{f}(x) \supset P_{f}(f(f(f(x))))\right\}$. For $S_{3}$, it is obvious that $\lambda_{d_{0}}(x)=\neg P_{f}(x), \lambda_{d_{1}}(x)=P_{f}(x)$, and $\lambda_{d_{2}}(x)=\neg P_{f}(x)$. The meaning of them under the interpretation differ from those of $S_{1}$.

The expansion of $S_{3}$ is $S_{4}=\left\{P_{c}, \neg P_{f c}, P_{f f}(f(c)), P_{f c} \supset \neg P_{f f}(c), P_{f f}(x) \supset\right.$ $\left.\neg P_{f f}(f(x)), P_{f c} \supset P_{f f}(f(f(c))), P_{f f}(x) \supset P_{f f}(f(f(f(x))))\right\}$. We take a contraction $S_{5} \quad$ of $S_{4}$, such that $S_{5}=\left\{P_{c}, \neg P_{f c}, P_{f f}(f(c)), P_{f f}(x) \supset \neg P_{f f}(f(x)), P_{f f}(x) \supset\right.$ $\left.P_{f f}(f(f(f(x))))\right\}$. For $S_{5}$ it is obvious that $\lambda_{d_{0}}(x)=\neg P_{f f}(x), \lambda_{d_{1}}(x)=\neg P_{f f}(x)$, and $\lambda_{d_{2}}(x)=P_{f f}(x)$. The meaning of them differ from those of $S_{1}$ and $S_{3}$.

The expansion of $S_{5}$ is $S_{6}=\left\{P_{c}, \neg P_{f c}, P_{f f f}(c), P_{f f c} \supset \neg P_{f f f}(f(c)), P_{f f f}(x) \supset\right.$ $\left.\neg P_{f f f}(f(x)), P_{f f c} \supset P_{f f f}(f(f(f(c)))), P_{f f f}(x) \supset P_{f f f}(f(f(f(x))))\right\}$. We set $S_{7}=S_{6}$. For $S_{7}$, it is obvious that $\lambda_{d_{0}}(x)=P_{f f f}(x), \lambda_{d_{1}}(x)=\neg P_{f f f}(x)$, and $\lambda_{d_{2}}(x)=\neg P_{f f f}(x)$. The meaning of them coincide with those of $S_{1}$. Therefore we can set a flat transformation $R$ from $\boldsymbol{L}_{P}\left(S_{5}\right)$ to $\boldsymbol{L}_{\boldsymbol{P}}\left(S_{5}\right)$ such that $P_{f f f}(x)^{R}=P(x), P_{c}=$ true, $P_{f c}=$ false, $P_{f f c}=$ false. The set $S_{7}{ }^{R}$ is $\{P(c), P(x) \supset \neg P(f(x)), P(x) \supset P(f(f(f(x))))\}$ and equals to $S_{1}$.

\section{§7. Discussions}

We have presented an unsatisfiability-satisfiability prover for the first order logic. This procedure terminates for theorems of first order logic by the well known fact. This property is the completeness in ordinary sense for theorem provers. Our main purpose in this paper is the treatment of non-theorems, and we have shown two classes for which the procedure terminates. One is shown in $\$ 6$ that is the class of formulas whose clausal form is satisfiable in some finite domain. Hence for the class of formulas whose decidability is shown by the reduction to finite domain, for example the class of formulas whose every predicate is monadic, the procedure works as a decision procedure. That is if a given formula is a theorem then the procedure detects it by the reason that it is a theorem, and if given one is not a theorem, this means that the formula is false in some finite domain. Consequently the procedure detects it by the result in $\S 6$.

The other is shown in $\S 5$ that is the class of sets of clauses which is 
satisfiable in a simple Herbrand Universe theory. This class is not so interesting in the current status, but at least it is not included in the class in the $\S 6$, for example the set $E$ of clauses in $\$ 5$ is not satisfiable in any finite domain.

For the future investigation for the effort to widen the detection of the satisfiability, there may be needed more techniques, and the target will be the extension of the results in $\S 5$.

We paid no effort on the optimization of the procedure in this paper. But of course this is important for the implementations, for example tautologies should be removed at once, and some propositional calculus of predicate variables can be permitted in configuration construction. The operations of quasicontractions are perhaps necessary, but this necessity is not shown in this paper. Also the difference between the elementary satisfactory configuration and the simple satisfactory configuration is not shown in this paper. The compatibility of the procedure with the resolution is not clear. Therefore improvement of efficiency in this direction may need some efforts.

\section{Acknowledgements}

The author thanks to Prof. S. Takasu who guided this study and gave many helpful discussions and comments, Prof. T. Tsuji and T. Mizutani for their many suggestions, and Prof. S. Igarashi of his supervising of this study. This work is supported in part by the Grant-in-Aid for the Scientific Research of Ministry of Education, Science and Culture (No. 62302005).

\section{References}

[1] Andrews, P. B., Theorem proving via general matings, J. ACM, 28 (1981), 193-214.

[2] Chang, C.L., Theorem proving with variable-constrained resolution, Information Science, 4 (1972), 217-231.

[3] Chang, C.L. and Lee, R.C., Symbolic logic and mechanical theorem proving, Academic Press, New York, 1973.

[4] Fribourg, L., A superposition oriented theorem prover, Theoretical Computer Science, 35 (1985), 129-164.

[5] Hosono, C., A method of theorem proving-a modification of the variable constrained resolution, Tensor, N.S., 46 (1987), 44-51.

[6] Plaisted, D. A., Theorem proving with abstraction, Artificial Intelligence, 16 (1981), $47-108$. 
